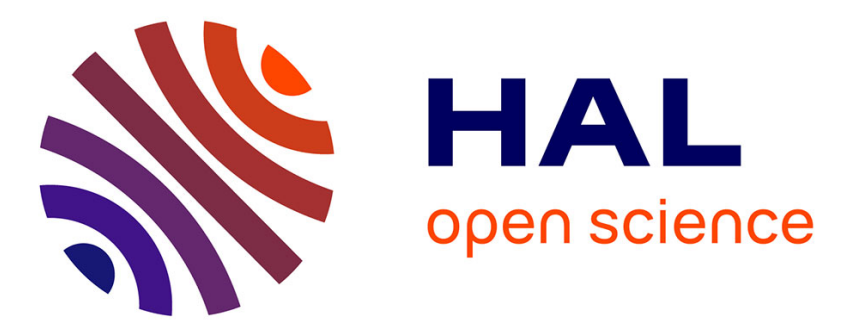

\title{
Origin of weak Fermi level pinning at the graphene/silicon interface
}

Courtin Jules, S. Tricot, G. Delhaye, Pascal Turban, B. Lépine, Jc Le Breton, Philippe Schieffer

\section{- To cite this version:}

Courtin Jules, S. Tricot, G. Delhaye, Pascal Turban, B. Lépine, et al.. Origin of weak Fermi level pinning at the graphene/silicon interface. Physical Review B, 2020, 102 (24), pp.245301. 10.1103/PhysRevB.102.245301 . hal-03081365

\section{HAL Id: hal-03081365 https://hal.science/hal-03081365}

Submitted on 18 Dec 2020

HAL is a multi-disciplinary open access archive for the deposit and dissemination of scientific research documents, whether they are published or not. The documents may come from teaching and research institutions in France or abroad, or from public or private research centers.
L'archive ouverte pluridisciplinaire HAL, est destinée au dépôt et à la diffusion de documents scientifiques de niveau recherche, publiés ou non, émanant des établissements d'enseignement et de recherche français ou étrangers, des laboratoires publics ou privés. 


\title{
Origin of Weak Fermi Level Pinning at the Graphene-Silicon
}

\section{Interface}

\author{
J. Courtin, S. Tricot, G. Delhaye, P. Turban, B. Lépine, J.-C. Le Breton, and P. Schieffer* \\ Univ Rennes, CNRS, IPR (Institut de Physique de Rennes)-UMR 6251, F-35000 Rennes, France
}

(Received 27 July 2020)

\begin{abstract}
The mechanisms governing the formation of Schottky barriers at graphene/hydrogenpassivated silicon interfaces where the graphene plays the role of a two-dimensional (2D) metal electrode have been investigated by means of x-ray photoemission spectroscopy and density functional theory (DFT) calculations. To control the graphene work function without altering neither the structure nor the band dispersion of graphene we used a method that consists in depositing small amounts of gold forming clusters on the graphene/hydrogenpassivated silicon system under ultra-high vacuum environment. We observe from experimental measurements that the Fermi level is mainly free from pinning at the graphene/hydrogen-silicon interface whereas for a semi-infinite metal on silicon the Fermi level is almost fully pinned. This alleviation of the Fermi level pinning observed with the graphene layer is explained by DFT calculations showing that the graphene and the semiconductor are decoupled and that the metal-induced gap states (MIGS) density at the silicon midgap at the interface is very low $\left(<5 \times 10^{10}\right.$ states $\left./ \mathrm{eV} / \mathrm{cm}^{2}\right)$. The important conclusion that stems from the DFT results analysis is that the low MIGS density at the semiconductor midgap is related to the $2 \mathrm{D}$ nature of the graphene layer. More precisely, the MIGS density is low owing to the lack of propagating states perpendicular to the graphene layer. This finding brings precious information to understand the mechanisms that govern the
\end{abstract}


formation and the electronic properties of Schottky barriers at 2D-metal/3D-semiconductor interfaces.

*Corresponding author.philippe.schieffer@univ-rennes1.fr 


\section{INTRODUCTION}

Graphene (Gr), a monolayer of carbon atoms arranged in a two-dimensional (2D) honeycomb lattice is a semimetal with linearly dispersing states at low carrier energies [1]. It stimulated tremendous research interest primarily owing to its high optical transparency $[2,3]$ and ultra-high charge carriers mobility [4] and appears as a promising material for future applications in electronics, optoelectronics, and photovoltaics [5-10]. More specifically, graphene has attracted intensive attention during the last years due to its remarkable physical properties and its compatibility with silicon technology. Different kind of devices based on graphene-silicon Schottky barriers were developed as Schottky junction solar cells [5,11,12], graphene diode sensors $[13,14]$ or photodetectors [13,15-17]. A graphene-silicon barristor was also proposed by Yang et al. [18] with a gate-controlled Schottky barrier. Its mode of operation takes advantage of the fact that the Fermi level within the graphene can be adjusted with a gate-voltage and that the Fermi level is unpinned at the graphene/silicon interface.

The key issue of the mode of operation of graphene-silicon-based devices lies in the control of the Schottky barrier height (SBH), the density of interface sates or the doping level in graphene. Here, the $\mathrm{SBH}$ is defined as the difference between the semiconductor conduction band edge and the Fermi level at the interface. Despite the large number of studies on the graphene/silicon system [19-22] the mechanisms governing the Schottky barrier formation and the degree of interface Fermi level pinning in this system are not well understood. In metal/silicon systems the SBH is well explained using the metal-induced gap states (MIGS) model [23] combined with the charge neutrality level concept [24,25]. MIGS, whose density in the semiconductor midgap is above $10^{14}$ states $/ \mathrm{eV} / \mathrm{cm}^{2}$ [26], are responsible for the Fermi level pinning at the metal/silicon interface. The Fermi level pinning was found to be close to the charge neutrality level of silicon which is $\sim 0.4 \mathrm{eV}$ above the valence band maximum (VBM) of silicon [24,27]. In contrast, the role played by the MIGS is much less 
clear when replacing the metal with a graphene layer. More specifically the Fermi level was found unpinned at the graphene/passivated-silicon interface $[18,28-30]$ indicating that the MIGS and the interface defect states density must be low (typically below $10^{12}$ states $/ \mathrm{eV} / \mathrm{cm}^{2}$ ). We expect that, with non-passivated silicon surfaces, Gr/silicon SBH is controlled by interface defect states. The question arising now is, why is the MIGS density so weak in the $\mathrm{Gr} /$ silicon system? In order to answer this question, we have combined theoretical and experimental approaches to study the SBH variations at the Gr/hydrogen-passivated silicon interface (noted as $\mathrm{Gr} / \mathrm{H}-\mathrm{Si}$ in the following) induced by the graphene work function changes. The silicon surfaces were passivated with hydrogen atoms forming $\mathrm{Si}-\mathrm{H}$ bonds [31-33] allowing to suppress the electrically active states density related to the surface silicon dangling bonds. In the experiments conducted with the barristor [18] the graphene chemical potential was controlled by adjusting the gate-voltage using a gate-insulator-graphene structure requiring to form an insulator/graphene interface. Here we have explored another method to continuously shift the chemical potential of graphene. By depositing small amounts $\left(\sim 10^{14}\right.$ atoms $\left./ \mathrm{cm}^{2}\right)$ of gold forming clusters on the $\mathrm{Gr} / \mathrm{H}-\mathrm{Si}$ system under ultra-high vacuum (UHV) environment we were able to manipulate the graphene work function as this was done in Ref. 34 without altering neither the structure nor the band dispersion of graphene.

From the density functional theory (DFT) calculations we find that the electronic properties of the free-standing graphene are not altered by the adsorption of gold adatoms, only the graphene chemical potential is modified leading to a holes-doped graphene layer. In addition, we observe that the Fermi level position movement away from the Dirac point induced by the $\mathrm{Au}$ adatoms adsorption on one side of graphene is accompanied by a similar change of the work function on the free-side of graphene. Using the core-level x-ray photoemission spectroscopy (XPS) it was then possible to follow the evolution of the $\mathrm{Gr} / \mathrm{H}-\mathrm{Si}$ $\mathrm{SBH}$ as a function of the graphene work function and to observe the absence of Fermi level 
pinning at the $\mathrm{Gr} / \mathrm{H}-\mathrm{Si}(001)$ interface. Our DFT theoretical works performed at $0 \mathrm{~K}$ and for an undoped semiconductor show that this behavior is explained by the fact that the graphene layer and the semiconductor are decoupled and that the MIGS density at the silicon midgap at the interface is low $\left(<5 \times 10^{10}\right.$ states $\left./ \mathrm{eV} / \mathrm{cm}^{2}\right)$. A very important conclusion that stems from our work is that the MIGS density at the semiconductor midgap is low owing to the twodimensional nature of the graphene layer. This can be easily understood if we consider that the electronic band structure of graphene is well defined in the plane whereas this twodimensional system does not have propagating states in the direction perpendicular to the layer.

\section{EXPERIMENTAL DETAILS}

Single layer graphene grown by chemical vapor deposition on copper was purchased from GrapheneaC. A $200 \mathrm{~nm}$ polymethyl methacrylate (PMMA) layer was spin-coated on the graphene/ $\mathrm{Cu}$ foil samples. After copper etching in a $\mathrm{FeCl}_{3}$ solution during 1-2 hours, the samples were rinsed several times in deionized water (resistivity of $\sim 18 \mathrm{M} \Omega . \mathrm{cm}$ ) and $\mathrm{HCl}$ (12\%) bathes. Double-side polished $n$-type $\mathrm{Si}(001)$ substrates (doped with phosphorus) with a doping concentration of $7 \times 10^{14} / \mathrm{cm}^{3}$ (determined by Hall effect measurements) were degreased in acetone and isopropyl alcohol followed by 30 minutes of UV-ozone cleaning leading to the formation of an approximately $1 \mathrm{~nm}$-thick $\mathrm{SiO}_{2}$ layer. The floating PMMAcoated graphene membranes were then transferred onto the hydrophilic $\mathrm{SiO}_{2}$ surfaces and air dried. PMMA was removed in acetone bath followed by isopropyl alcohol rinse and $\mathrm{N}_{2}$ blow dry. Samples were then dipped in $\mathrm{HF}(2 \%)$ for 1 min to remove the $\mathrm{SiO}_{2}$ layer and rinsed with deionized water for $30 \mathrm{~s}$ to passivate the silicon surface with hydrogen. The $\mathrm{Gr} / \mathrm{H}-\mathrm{Si}(001)$ samples were then annealed at $300^{\circ} \mathrm{C}$ during $3 \mathrm{~h}$ below $10^{-7} \mathrm{~Pa}$ to eliminate the PMMA residues. Finally, the samples were dipped in $\mathrm{HF}(2 \%)$ a second time followed by a deionized water rinse just before their introduction in our UHV multichamber system (with a base 
pressure below $\left.10^{-8} \mathrm{~Pa}\right)$. Au was sequentially deposited onto $\mathrm{Gr} / \mathrm{H}-\mathrm{Si}(001)$ samples in a UHV interconnected chamber from an effusion cell at a rate of $0.4 \AA / \mathrm{min}$, below $5 \times 10^{-8} \mathrm{~Pa}$ and on substrates maintained at room temperature. Photoemission measurements were carried out using x-ray photoelectron spectroscopy with $\mathrm{Mg} K \alpha$ as source and the spectra were recorded at normal emission. The kinetic energy of the emitted electrons was measured by employing a hemispherical analyzer (Omicron EA125) with a five-channel detection system, an energy resolution better than $0.9 \mathrm{eV}$, and an angular resolution of $\sim 1^{\circ}$. The $\mathrm{Au} 4 f_{7 / 2}$ peak position from a thick gold sample, assumed to be $84.00 \mathrm{eV}$ binding energy (BE) [35], was taken as a reference in order to position the Fermi level. The Si $2 p$ and C $1 s$ core-levels were monitored as a function of gold thickness. In our experiments, the binding energy was measured with respect to the Fermi level of the sample.

\section{COMPUTATIONAL DETAILS}

In the present work, DFT calculations were performed with the GPAW code $[36,37]$ on various systems, namely, gold adatoms on free-standing graphene, graphene and gold layer on $\mathrm{H}-\mathrm{Si}(111)$ surface and metal adatoms on $\mathrm{Gr} / \mathrm{H}-\mathrm{Si}(111)$ structure. For the sake of simplicity we only considered in our computations unreconstructed H-Si(111) surfaces whose the sixfold symmetry is the same as that of the hexagonal lattice of graphene. We expect that a study on $\mathrm{Si}(001)$ surfaces leads to same conclusions as those drawn here. This is justified because Schottky barriers are primarily insensitive to crystallographic orientation of semiconductor surfaces [27]. DFT calculations were carried out in the generalized gradient approximation (GGA) using the Perdew-Burke-Ernzerhof (PBE) exchange-correlation functional [38] on a Monkhorst-Pack $k$-point grid [39] and a plane wave basis kinetic energy cutoff of $400 \mathrm{eV}$. All structures were relaxed until the maximum force acting on each atom was less than 0.05 eV/A. The vacuum region between adjacent slabs was set to $\sim 20 \AA$ and dipole correction was applied in order to calculate the work functions. The charges of the different atoms have been 
obtained through a Bader analysis [40]. The calculation for free-standing graphene with or without $\mathrm{Au}$ adatoms were performed using $12 \times 12 \times 1 k$-point meshes with a graphene slab modeled with a $3 \times 3 \times 1$ supercell which contained 18 carbon atoms. The DFT-optimized graphene lattice parameter $\left(a_{\mathrm{Gr}}\right)$ is $2.46 \AA$ identical to the experimental value. The $\mathrm{Si}(111)$ based slabs (the slabs will be shown later in the paper) were modeled with 12 atomic layers and an in-plane unit cell parameter set to $3.87 \AA$, derived from the predicted lattice parameter of bulk Si (5.47 $\AA$ ). They were passivated with a hydrogen monolayer on both sides (unless specified otherwise). In order to minimize the lattice mismatch, $\mathrm{Gr} / \mathrm{H}-\mathrm{Si}(111)$ slabs were modeled with a single carbon layer with $3 \times 3 \times 1$ and $2 \times 2 \times 1$ supercells for graphene layer and $\mathrm{Si}$, respectively. The calculations and Brillouin zone sampling integration was performed using $2 \times 2 \times 1$ Monkhorst-Pack meshes for the DFT-optimization and $12 \times 12 \times 1$ for the density of states calculation. With metal adatoms on $\mathrm{Gr} / \mathrm{H}-\mathrm{Si}(111)$ the computational parameters were taken identical to those used for $\mathrm{Gr} /$ silicon contacts. In our calculations the adsorption of adatoms such as $\mathrm{Au}, \mathrm{Cu}$ and $\mathrm{Ag}$ were considered in order to change the doping (nature and concentration) of graphene. Finally, the $\mathrm{Au} / \mathrm{H}-\mathrm{Si}(111)$ slabs were modeled with four $\mathrm{Au}(111)$ atomic layers. We chose $4 \times 4 \times 1$ and $3 \times 3 \times 1$ supercells for $\mathrm{Au}(111)$ and Si(111), respectively, to lessen the lattice mismatch. The calculations and Brillouin zone integration was performed using $2 \times 2 \times 1$ Monkhorst-Pack meshes for the DFT-optimization and $6 \times 6 \times 1$ for the density of states and work function calculations.

\section{RESULTS AND DISCUSSION}

\section{A. Photoemission results}

Figure 1(a) presents Si $2 p$ core-level spectra (normalized to their own maximum value and vertically shifted for clarity) taken at normal emission for different Au thicknesses deposited onto $\mathrm{Gr} / \mathrm{H}-\mathrm{Si}(001)$ at room temperature. The spectra were decomposed after background subtraction with a least-square procedure using a Lorentzian convoluted with a 
Gaussian to represent each spin-orbit component of the Si $2 p$ core-level. In the fitting procedure the spin-orbit splitting of the $\mathrm{Si} 2 p$ core-level was fixed at $0.60 \mathrm{eV}$ and the branching ratio was taken to be the statistical value of 2 . The spectra whose the line shape does not change upon metal deposition are dominated by the Si $2 p_{3 / 2}$ and $\mathrm{Si} 2 p_{1 / 2}$ core-level contributions which appear as a single feature in the photoemission spectrum. As the $\mathrm{Au}$ thickness increases we observe a small variation in the Si core-level binding energy $(\sim 0.1 \mathrm{eV})$ that is caused by an upward band bending change in Si. We have to include an additional component located $\sim 1.0 \mathrm{eV}$ higher than the binding energy of the main component for every Au thicknesses to improve the fits. This component (labelled $\mathrm{Si}^{1+}$ ) is related to silicon atoms in an oxidation state +1 suggesting that some silicon atoms are involved in a $\mathrm{Si}_{2} \mathrm{O}$ phase at the silicon surface [41]. From the quantitative analysis of photoemission intensities we find that these $\mathrm{Si}_{2} \mathrm{O}$ patches occupy less than $10 \%$ of the silicon surface. A similar amount of oxides was observed for hydrogen-passivated $\mathrm{Si}(001)$ surfaces [29].

From our XPS experiments we can obtain the position of the silicon conduction band edge at the $\mathrm{Gr} / \mathrm{H}-\mathrm{Si}$ interface relative to the position of the Fermi level that allows to determine the value of the SBH and the band bending in silicon. These results are derived from the Si $2 p_{3 / 2}$ core-level binding energy of the main component using a silicon bandgap of $1.121 \mathrm{eV}$ [42], an energy separation of $98.74 \mathrm{eV}$ between the VBM and the Si $2 p_{3 / 2}$ core-level [43] and a doping concentration of $7 \times 10^{14} / \mathrm{cm}^{3}$ (the Fermi level in bulk is $0.28 \pm 0.01 \mathrm{eV}$ below the conduction band minimum (CBM)). The binding energy of the Si $2 p_{3 / 2}$ core-level obtained from our fitting procedure for the bare $\mathrm{Gr} / \mathrm{H}-\mathrm{Si}(001)$ surface is $99.62 \pm 0.03 \mathrm{eV}$. It is important to note that for photoelectrons emitted from light elements with a high kinetic energy (typically higher than $0.5 \mathrm{keV}$ ), the recoil effect causes an apparent and significant increase of the binding energy of the core-level [44]. The recoil energy is estimated to be 22 meV for the $\mathrm{Mg} K \alpha$ excited Si $2 p$ core-level (the kinetic energy of photoelectrons is $\sim 1.15$ 
$\mathrm{keV})$. Taking into account this effect in our data analysis we obtain a SBH value of $0.26 \pm$ $0.04 \mathrm{eV}$. Thus, within the limits of experimental uncertainties this indicates that the semiconductor is very close to the flat band situation for the bare $\mathrm{Gr} / \mathrm{H}-\mathrm{Si}(001)$ structure. Figure 1(b) gives the evolution of the $\mathrm{SBH}\left(\phi_{B n}\right)$ as a function of the Au thickness. It can be seen that the SBH increases as a function of the Au thickness up to $0.36 \pm 0.04 \mathrm{eV}$ for a gold thickness of $1.0 \AA$. These results show that the SBH can be continuously modified by a simple metal adsorption on the graphene surface.

Figure 2(a) shows the $\mathrm{Mg} K \alpha$ excited $\mathrm{C} 1 s$ core-level photoemission spectra (normalized to their own maximum value and vertically shifted for clarity) at normal emission for several Au thicknesses and for $\mathrm{Gr} / \mathrm{H}-\mathrm{Si}(001)$ before gold deposition. The kinetic energy of photoelectrons is $\sim 0.96 \mathrm{keV}$. The experimental spectra were decomposed after background subtraction with a least-square procedure using a Lorentzian convoluted with a Gaussian to represent each component of the $\mathrm{C} 1 s$ core-level. Before gold deposition, the spectrum is dominated by a component located at $284.60 \pm 0.03 \mathrm{eV}$ related to carbon atoms with $\mathrm{sp} 2$ hybridization that are connected in a honeycomb lattice structure. To obtain satisfactory fits we had to include an additional component located at $\sim 0.50 \mathrm{eV}$ higher binding energy than the main component corresponding to a sp3 hybridized carbon [45]. Contribution from PMMA related peaks $(\mathrm{C} 1, \mathrm{C} 2, \mathrm{C} 3$ and $\mathrm{C} 4)$ in the binding energy range $1.3-4.8 \mathrm{eV}$ higher than the main component $[46,47]$ have to be introduced as well showing that PMMA residues were not completely removed through our cleaning procedure. These components contribute only to $\sim 10 \%$ of the total intensity of the $\mathrm{C} 1 s$ core-level spectrum. The $\mathrm{C} 1 s$ binding energy of the sp2 component for a free-standing and undoped graphene layer (where the Fermi level is assumed to coincide with the Dirac point) was found to be $284.85 \mathrm{eV}$ [48] with an excitation source of $380 \mathrm{eV}$. The binding energy correction related to the recoil effect for a kinetic energy of $\sim 0.96 \mathrm{keV}$ is estimated to be $44 \mathrm{meV}$. Taking into account this effect in our 
data analysis we estimate the Fermi level position in graphene for the $\mathrm{Gr} / \mathrm{H}-\mathrm{Si}(001)$ sample to be $\sim 0.29 \pm 0.04 \mathrm{eV}$ below the Dirac point showing that the graphene is $p$-doped.

The line shape of the C $1 s$ core-level spectra does not change with increasing the gold thickness, only a slight rigid shift of a fraction of $\mathrm{eV}$ is detected [Fig. 2(b)]. It was predicted from first-principles DFT calculations [49-51] and experimentally shown using highresolution angle-resolved photoemission [52] that contacting graphene with a gold layer does not modify the band dispersion of graphene but induces a $p$-doping of graphene. We propose therefore that the variation of the $\mathrm{C} 1 s$ core-level binding energy is primarily induced by the shift of the Fermi level relative to the Dirac point over the entire sequence of metal deposition. This point will be considered in detail in the following section using DFT calculation. It can be noted that the deposition of small amounts of gold on graphene layers at room temperature leads to the formation of gold clusters on graphene surfaces as demonstrated by atomic force microscopy [53,54] and scanning tunneling microscopy [55]. We can therefore expect that the graphene doping changes induced by the metal deposition is caused by electron transfer from the graphene sheet to the gold clusters.

Figure 3 shows the evolution of the $\operatorname{Si} 2 p_{3 / 2}$ core-level binding energy as a function of the $\mathrm{C} 1 s$ core-level binding energy for various $\mathrm{Au}$ thicknesses. The experimental points are aligned on a straight line of slope $\sim 0.9$. We will see in the following section that this slope characterizes the degree of Fermi level pinning at the metal/semiconductor interface. When the Fermi level is not pinned at the interface the slope is equal to unity corresponding to the Schottky-Mott limit. This happens when the density of interface states in the semiconductor bandgap at the interface is low (typically below $10^{12}$ states $/ \mathrm{cm}^{2}$ ). In contrast, when the Fermi level is strongly pinned the slope is close to zero corresponding to a situation where the interface states density is higher than $10^{14}$ states $/ \mathrm{cm}^{2}$. In this case the SBH value is independent of the metal work function. Thus we can conclude from our data that at the $\mathrm{Gr} / \mathrm{H}-$ 
$\mathrm{Si}(001)$ interface, the Fermi level is mainly free from pinning and that it is possible to control the SBH by a metal adsorption on a graphene surface. This behavior is very similar to the observed one with a three-terminal-based device in which the SBH is also tuned but using a gate-voltage control [18]. This demonstrates that the density of interface states at the $\mathrm{Gr} /$ hydrogen-passivated silicon interface is low. In the following section we will explain this peculiarity using DFT calculations.

\section{B. DFT results}

We performed first-principles DFT calculations on adsorbed gold atoms on a graphene surface to deeper understand the impact of such adsorption on the electronic properties of the underlying carbon layer. In particular, we are interested in the doping changes and work function modifications of the graphene sheet induced by metal adsorption. Three different sites for the Au adsorption on graphene surfaces were considered: top, hollow and bridge sites [Fig. 4]. The adsorption energy is defined as follows:

$$
E_{\mathrm{ads}}=E_{\mathrm{Gr}}+E_{\mathrm{Au}}-E_{\mathrm{Gr}, \mathrm{Au}},
$$

where $E_{\mathrm{Gr}}$ is the total energy of an isolated graphene constituted by $3 \times 3$ graphene unit cells (18 C atoms), $E_{\mathrm{Au}}$ is the energy of an isolated gold atom and $E_{\mathrm{Gr}, \mathrm{Au}}$ is the energy of the system where an $\mathrm{Au}$ adatom is adsorbed per supercell on the graphene surface. A positive value for $E_{\text {ads }}$ means a favorable adsorption. The adsorption geometry is obtained from the positions of atoms after relaxation. The equilibrium distance $\left(d_{\mathrm{M}-\mathrm{Gr}}\right)$ between the Au adatom and graphene sheet is defined as the difference in $z$ coordinate $(z$ direction is perpendicular to graphene) of the adatom and the average of the $z$ coordinate of the $\mathrm{C}$ atoms in the graphene layer. The charge transferred $\left(Q_{\mathrm{Au}}\right)$ from the graphene to the Au atom was obtained through a Bader analysis [40]. A negative charge transfer means a transfer of electrons from the graphene sheet to $\mathrm{Au}$ atoms. The values obtained from our calculations for the adsorption 
energy, the structural geometry and the charge transferred for the three configurations are reported in Table I. Our data are close to previous theoretical results [56-59] and show that $\mathrm{Au}$ has small adsorption energy which depends little on the adsorption sites. Due to their high electronegativity gold atoms are negatively charged after adsorption on graphene. The charge transfer during the adsorption phase of gold atoms can therefore induce a $p$-doping of the graphene as this was observed experimentally $[60,61]$ and theoretically $[57,58,62-65]$.

Figure 5 shows the calculated projected density of states (PDOS) on atomic orbital of carbon atoms for relaxed structures with Au atoms on the top, bridge and hollow sites of graphene and for an isolated graphene. The PDOS's were computed with $12 \times 12 \times 1 k$-point meshes and a Gaussian smearing of $0.2 \mathrm{eV}$. The energy is relative to the position of the Fermi level $\left(E_{F}\right)$. As expected for a free-standing and undoped (pristine) graphene the Fermi level coincides with the Dirac crossing point where the PDOS is close to zero. The shape of the PDOS's calculated for Au adsorbed on the bridge and hollow sites are very similar to that of pristine graphene. However, after the Au adsorption the PDOS's are shifted by $0.43 \mathrm{eV}$ toward higher energies. The Fermi level is then $0.43 \mathrm{eV}$ below the Dirac crossing point confirming that the graphene is $p$-doped for Au adsorption on bridge or hollow sites.

The PDOS of graphene is slightly changed when Au atoms are adsorbed on top sites. $\mathrm{Au}$ adsorption causes a global shift of $0.17 \mathrm{eV}$ toward higher energies of the PDOS and the appearance of two prominent peaks in the PDOS at -1.4 and $0.0 \mathrm{eV}$. As discussed in Refs. 58 and 66 the structure at $-1.4(0.0) \mathrm{eV}$ results from the overlap of the $5 d(6 s)$ orbitals of the gold atom and the orbitals of the nearest carbon atoms. The presence of the Au adatoms moreover leads to partially empty the graphene valence band. This indicates that the graphene layer must also be $p$-doped as deduced from the sign of the charge transfer (as obtained through Bader analysis) between the graphene and the Au adatoms. 
To summarize, our theoretical results demonstrate that the adsorption of gold atoms on graphene causes a $p$-doping of the graphene sheet while preserving its electronic structure. The same conclusion was drawn from theoretical works for graphene sheets contacted with an Au substrate $[49,67]$ or Au clusters $[58,68]$.

Let us now discuss the work function $\left(\Phi_{\mathrm{Gr}}\right)$ values of graphene with and without adsorbed Au atoms. The graphene sheet lies in the $x y$-plane and $z$ is perpendicular to it. In Fig. 6 is shown the $x y$-planar averaged electrostatic potential along the $z$ direction for the relaxed structure of $\mathrm{Au}$ atoms on the top site of graphene and for pristine graphene. $\Phi_{\mathrm{Gr}}$ is the difference between the position of the vacuum level and the Fermi level $\left(E_{F}\right)$. The vacuum level is obtained from the averaged electrostatic potential far enough away from the graphene in regions where the potential remains constant over several angstroms. The vertical dashed line indicates the position of the graphene layer and the single vertical arrow gives the equilibrium position of the gold adatoms. For the pristine graphene the vacuum level energy is the same on both sides of the graphene layer. The value of $\Phi_{\mathrm{Gr}}$ deduced from the averaged electrostatic potential is $4.23 \mathrm{eV}$, a value close to that calculated by others groups using the PBE functional [57,69-72].

With $\mathrm{Au}$ adatoms, the vacuum level position relative to $E_{\mathrm{F}}$ differs on both sides of the structure. The Au adsorption induces an increase of the work function of $0.17 \mathrm{eV}$ on the free graphene surface side (left) and of $1.0 \mathrm{eV}$ on the side on which sit the Au atoms (right). Such an increase of the work function on the right side was theoretically observed by Chan et al. [57]. It can be ascribed to the appearance of a surface dipole moment perpendicular to the surface related to the charge transfer from graphene to Au adatoms and from the emptying of graphene valence states. However, because of the significant covalent character of the Au-C bonds [57] the precise origin of the work function change on the right side of the system induced by the Au adsorption still remains unclear. 
On the free graphene side (left side) we observe that the work function variation induced by $\mathrm{Au}$ adsorption is very close to the variation of the energy difference $E_{D F}=E_{D}-E_{F}$, with $E_{D}$ the Dirac point energy in the graphene band structure. In Fig. 7 is plotted the variation of the work function on the free graphene side $\left(\Phi_{\mathrm{Gr}}\right)$ as a function of $E_{D F}$ for $\mathrm{Au}$ in the three configurations considered in the present work. In the figure are also plotted the DFT results obtained for two Au adatoms occupying equivalent sites in the supercell and positioned to maximize the distance between them. The curve is linear with a slope of 0.96 (close to 1.0) showing that the work function variation is primarily controlled by the Fermi level position changes. This result suggests that the carbon atoms reorganization induced by the adsorption of $\mathrm{Au}$ atoms plays a negligible role on the value of the work function on the free graphene side.

The interesting point emerging from our analysis is that, the variation of the Fermi level position (relative to that of the Dirac point) induced by the Au adsorption gives directly the variation of the work function on the free graphene side following the relation:

$$
\frac{d \Phi_{\mathrm{Gr}}}{d E_{F}} \approx-1.0
$$

We have performed first-principles DFT calculations to investigate the electronic properties of $\mathrm{Gr} / \mathrm{H}-\mathrm{Si}(111)$ and $\mathrm{Au} / \mathrm{H}-\mathrm{Si}(111)$ interfaces. Comparing these two systems will allow us to better understand the role played by the MIGS at the $\mathrm{Gr} / \mathrm{H}-\mathrm{Si}(111)$ interface. The computational details are given in Sec. III of the present paper. In our experimental protocol the silicon substrates were passivated with hydrogen using HF etching after the graphene transfer. The surface dangling bonds of the silicon substrate are passivated with hydrogen atoms by forming $\mathrm{Si}-\mathrm{H}$ bonds [31-33] allowing to reduce the electrically active surface states density typically below $2 \times 10^{10}$ states $/ \mathrm{eV} / \mathrm{cm}^{2}[29,73]$. The $\mathrm{Si}(111)$ surface is fully covered with hydrogen atoms which are placed on the top position of surface silicon atoms on both 
sides of the slab which includes 12 atomic plane of silicon [Fig. 8]. For the relaxed structure we find $\mathrm{Si}-\mathrm{H}$ bond lengths of $1.51 \AA$ A close to the values theoretically obtained by other groups [74-76]. In Fig. 8 the interplanar spacing between the different adjacent planes have been added.

In Fig. 9(a) is shown the optimized graphene/H-Si(111) structure obtained by the DFT calculations. In our calculations the graphene lattice was $4.9 \%$ stretched to accommodate the lattice mismatch with the $\mathrm{Si}(111)$ surface lattice. The silicon in-plane lattice parameter was then fixed to $3.87 \AA$ and the graphene lattice parameter after stretching was set to $2.58 \AA$. No wavy/buckled structures in the graphene sheet were formed during the optimizing process and an equilibrium distance of $3.11 \AA$ between the graphene sheet and the hydrogen plane was calculated. Contacting graphene with $\mathrm{H}-\mathrm{Si}(111)$ surfaces causes only little changes on the structure of the silicon slab and the graphene sheet as already found by Dang et al. [77]. Considering the accuracy of our DFT calculations we observe that the distance between two successive Si planes within the slab and the $\mathrm{Si}-\mathrm{H}$ bond lengths are negligibly changed. A very low electron transfer (below $2 \times 10^{11}$ electrons $/ \mathrm{cm}^{2}$ as obtained through Bader analysis) from the silicon-based structure to the graphene sheet occurs when contacting graphene with H$\operatorname{Si}(111)$. The electronic structure of graphene is preserved after contact as shown by the graphene PDOS's plotted in Fig. 9(b). Indeed it can be seen that the shape of the PDOS of Gr on $\mathrm{H}-\mathrm{Si}$ surface is very similar to that of the free-standing graphene calculated for graphene with the same lattice parameter of $2.58 \AA$. We have checked that this lattice expansion does not cause significant changes on the electronic properties of the free-standing graphene. We observe also in Fig. 9(b) that the Fermi level is $0.07 \mathrm{eV}$ above the Dirac point that shows that the initially undoped graphene becomes $n$-doped when contacted with the H-Si(111) surface which is consistent with the sign of charge transfer between the graphene sheet and the Sibased structure. The large distance between the graphene layer and the hydrogen-passivated 
silicon surface as well as the absence of alteration of the electronic properties of the graphene layer by the semiconductor show that the graphene layer is decoupled from the semiconductor substrate.

Let us now discuss our DFT calculation results for the $\mathrm{Au} / \mathrm{H}-\mathrm{Si}(111)$ interface. Similar DFT computations were conducted with $\mathrm{Ag}$ and $\mathrm{Al} / \mathrm{H}-\mathrm{Si}(111)$ [76] and $\mathrm{Al} / \mathrm{H}-\mathrm{Si}(100)$ [78] systems in order to specifically study the relation between the MIGS properties and the Fermi level pinning. Figure 10 shows the structural model used in the simulation after DFT optimization. We consider $4 \times 4 \times 1$ and $3 \times 3 \times 1$ supercells for $\mathrm{Au}(111)$ and $\mathrm{Si}(111)$, respectively. The Au lattice was then $1.2 \%$ stretched to accommodate the lattice mismatch with the $\operatorname{Si}(111)$ surface lattice. When the $\mathrm{Au}$ is contacted with the semiconductor structure the Si-H distance $\left(d_{\mathrm{H}-\mathrm{Si}}\right)$ slightly increases from 1.51 to $1.53 \AA$ while the variations of the silicon interplanar distances along the $z$ direction are less than $1.5 \%$. A close inspection of the structure shows that the Au interface layer has a slight corrugation with a difference in height of $\sim 0.2 \AA$ between the lowest and highest atom in the Au interface layer. We obtained an equilibrium average distance in $z$ coordinate between the position of the gold atoms in the interface plane and the hydrogen plane (first silicon layer) of 2.35 (3.88) $\AA$.

Figure 11(a) shows the layer-resolved projected density of states on silicon atomic orbitals (the atomic layer numbers are defined in Fig. 8) for the $\mathrm{Au} / \mathrm{H}-\mathrm{Si}(111)$ contact. The PDOS's were computed with $6 \times 6 \times 1 k$-point meshes and a Gaussian smearing of $0.2 \mathrm{eV}$. The silicon bandgap obtained in our calculation is $\sim 0.7 \mathrm{eV}$. This value is much smaller than the experimental value of $1.2 \mathrm{eV}$ [79]. This underestimation of the bandgap is an inherent and a well-known error in the GGA or local density approximation (LDA) approach [80,81], however, this fact has no impact on our conclusions.

Our DFT calculations with bare H-Si(111) slabs do not show any states within the silicon bandgap demonstrating that the hydrogen passivation completely suppresses the 
surface states in the silicon bandgap as this was found by Sajjad et al. [76]. We observe in Fig. 11(a) that contacting Au with H-Si(111) causes the appearance of a non-null DOS in the semiconductor bandgap in the first layers below the $\mathrm{Au} / \mathrm{H}-\mathrm{Si}(111)$ interface. These gap states, so-called metal-induced gap states in semiconductor bandgap, are induced by free-electrons metal states that penetrate into the semiconductor within the first silicon layers below the interface with an exponential decay [23]. In order to obtain the exponential decay length of the MIGS in the silicon we have plotted in Fig 11(b) in semilogarithmic scale the PDOS magnitude at the midgap (PDOS $\left.{ }_{\text {midgap }}\right)$ as a function of the depth in silicon $\left(d_{\mathrm{Si}}\right)$ relative to the first silicon layer. The curve is linear and the least-square fit of the data produces an exponential decay length $\left(\lambda_{\text {MIGS }}\right)$ of $\sim 0.27 \mathrm{~nm}$ consistent with the values of literature $[24,26,82]$.

The MIGS model [23] combined with the charge neutrality level concept [24,25] provides a solid ground to predict the chemical trends of the Schottky barrier height in metal/semiconductor contacts [83]. In order to analyze the degree of the Fermi level pinning at the metal/semiconductor interface it is convenient to introduce the semi-empirical parameter $S$ [84], called the slope parameter, which can be defined by the following relation:

$$
S=\frac{d \phi_{B n}}{d \Phi_{m}},
$$

with $\phi_{B n}$ the Schottky barrier height for an $n$-type semiconductor and $\Phi_{m}$ the metal work function. In the Schottky-Mott limit, corresponding to the alignment of the metal and semiconductor vacuum levels, the slope parameter approaches its maximum value of 1 . The Fermi level is then unpinned and there is negligible charge transfer between the metal and the semiconductor. This approach is valid for not-too-heavily doped semiconductors [84]. When the Fermi level is strongly pinned, the Schottky barrier height is independent of the metal work function and the $S$ parameter approaches 0 (Bardeen limit). 
Cowley and Sze [84] proposed in a phenomenological model, with a simple expression for the slope parameter. They considered an interfacial layer of thickness $\delta_{\text {int }}$ between the metal and the semiconductor and a constant density of interface states whose properties only depend on the semiconductor properties. The slope parameter can be expressed as follows:

$$
S=\frac{1}{1+\frac{q^{2} D_{\mathrm{is}} \delta_{\mathrm{int}}}{\varepsilon_{\mathrm{int}} \varepsilon_{0}}},
$$

with $q$ the elementary charge, $D_{\text {is }}$ the interface state density, $\varepsilon_{\text {int }}$ the relative dielectric constant of the interfacial layer and $\varepsilon_{0}$ the permittivity of vacuum. Louie et al. $[26,85]$ developed a more elaborated approach introducing the MIGS density at the semiconductor midgap ( $D_{\text {MIGS }}$ ) and proposed the following expression for the $S$ parameter:

$$
S=\frac{1}{1+\frac{q^{2} D_{\mathrm{MIGS}} \delta_{\mathrm{eff}}}{\varepsilon_{0}}},
$$

with $\delta_{\text {eff }}$ the effective distance between the negative charge placed in the wave function tail within the semiconductor and the positive charge left behind in the metal. The effective distance $\delta_{\text {eff }}$ is the sum of $\lambda_{\text {MIGS }}$ divided by the relative dielectric constant $\left(\varepsilon_{\mathrm{sc}}\right)$ of the semiconductor at the interface plus the metal Thomas-Fermi screening length $\left(\delta_{m}\right)$ which is typically of $0.5 \AA ̊$. More precisely $\varepsilon_{\mathrm{sc}}$ is the relative dielectric constant for screening potential fluctuations over a distance of the order of $\lambda_{\text {MIGS }}$ (Louie et al. [26,85] obtained $\varepsilon_{\mathrm{sc}}=2$, a value that we will take in the calculation of $S$ parameter). In the model, MIGS are assumed to have a constant density across the semiconductor bandgap.

In the present work we have calculated the $D_{\text {MIGS }}$ quantity by integrating the layer-bylayer silicon PDOS over the whole slab. Let us start the discussion with the slope parameter 
for an $\mathrm{Au} / \mathrm{Si}(111)$ intimate contact without interface hydrogen. The DFT calculations and the structural optimization were performed with the same supercell as for the $\mathrm{Au} / \mathrm{H}-\mathrm{Si}(111)$ system. We have obtained from the DFT calculations a MIGS density of $2.4 \times 10^{14} / \mathrm{cm}^{2} / \mathrm{eV}$ comparable with the data available in the literature $[24,26,82]$. The slope parameter $S$ calculated using Eq. (5) is $\sim 0.1$ indicating that the Fermi level is strongly pinned at the interface approaching the limit of Bardeen. This result is in close agreement with experimental results [27] from which a slope parameter of 0.1 was found. For the $\mathrm{Au} / \mathrm{H}-$ $\mathrm{Si}(111)$ contact we obtain a MIGS density of $3.7 \times 10^{13} / \mathrm{eV} / \mathrm{cm}^{2}$ giving a slope parameter of 0.4. The intercalation of hydrogen layer therefore causes an alleviation of the Fermi level pinning. In our calculations we have taken into account the presence of hydrogen by introducing the hydrogen layer thickness $\delta_{H}$ related to the hydrogen layer in the effective distance $\delta_{\text {eff }}$. The quantity $\delta_{H}$ was set to twice the covalent radius $(\sim 0.37 \AA)$ of hydrogen. Table II reports our results on the structural parameter of the interface, the MIGS characteristics, and the $S$ parameter for the different considered structures.

Let us now focus on the electronic properties of the $\mathrm{Gr} / \mathrm{H}-\mathrm{Si}(111)$ interface. It may be recalled that contacting graphene with $\mathrm{H}-\mathrm{Si}(111)$ surface does not alter the electronic properties of the graphene, only a slight rigid shift in energy of the carbon PDOS is observed [Fig. 9(b)]. The Fig. 12(a) shows the layer-resolved silicon PDOS for the Gr/H-Si(111) contact. One can see that states are present in the semiconductor bandgap (in the energy range 0-0.7 eV). The gap-states density is about 3 orders of magnitude lower than that observed for the $\mathrm{Au} / \mathrm{H}-\mathrm{Si}(111)$ contact. We estimate the MIGS density at the midgap of silicon around 4.3 $\times 10^{10}$ states $/ \mathrm{eV} / \mathrm{cm}^{2}$. The exponential decay length of the MIGS is around $0.36 \mathrm{~nm}$, slightly higher than that obtained with $\mathrm{Au} / \mathrm{H}-\mathrm{Si}(111)$ contact [Fig. 12(b)]. The curve shows oscillations in semilogaritmic representation that we are not able to interpret for now. 
The MIGS density at the semiconductor midgap is considerably lower than the density of states in the graphene except in the energy range of $\sim \pm 10 \mathrm{meV}$ around the Dirac crossing point [86]. This means that, when a graphene sheet and a H-Si(111) surface are brought into contact, the charge transfer occurring between the two materials will cause a negligible Fermi level shift (in absolute value $<20 \mathrm{meV}$ ) in the graphene sheet (we assume that the semiconductor remains in depletion regime). Hence, we propose that the relation $S=\mathrm{d} \phi_{B n} / \mathrm{d} \Phi_{m}$ which characterizes the degree of Fermi level pinning at metal/semiconductor interface remains valid for the $\mathrm{Gr} / \mathrm{H}-\mathrm{Si}(111)$ system, with $\Phi_{m}$ the work function of graphene. We will see below that the work function of graphene that must be considered here can be directly related to the Fermi level position in graphene.

The calculation of the $S$ parameter for $\mathrm{Gr} / \mathrm{H}-\mathrm{Si}(111)$ using the Eq. (5) requires to know the screening length $\delta_{m}$ in graphene that depends on the DOS at the Fermi level. It follows that $\delta_{m}$ must change with the carrier concentration in graphene as discussed in Refs. [87-89]. In particular, it was shown through theoretical works on the interlayer charge screening in few layers graphene structures that $\delta_{m}$ typically ranges from 0.06 to $0.4 \mathrm{~nm}$ [89] for carrier concentrations between $10^{16} / \mathrm{cm}^{2}$ and $10^{10} / \mathrm{cm}^{2}$. We have performed our calculations varying $\delta_{m}$ within the range $0.06-0.4 \mathrm{~nm}$ and found a slope parameter of 1.0. This result shows that the $\mathrm{Gr} / \mathrm{H}-\mathrm{Si}(111)$ system yields an ideal Schottky-Mott behavior.

In the present work we are interested in the Fermi level shift at the $\mathrm{Gr} / \mathrm{H}-\mathrm{Si}$ interface as a function of the graphene doping induced by the simple adsorption of $\mathrm{Au}$ on graphene. $\mathrm{We}$ have shown that the work function on the free graphene side (the side opposite to that on which the adatoms are deposited) is modified by the metal adsorption. In addition, we have demonstrated that the variation of the graphene Fermi level position relative to that of the Dirac point induced by the $\mathrm{Au}$ adsorption is directly related to the variation of the work function on the free graphene side through Eq. (2). As the graphene transfer on the 
semiconductor surface does not significantly modify the doping or the electronic structure of the graphene sheet we propose that the slope parameter for the adsorbate/Gr/H-Si structures can be directly deduced from the following relation:

$$
S \approx-\frac{d \phi_{B n}}{d E_{F}} .
$$

We have performed a complementary DFT-based study on the Schottky barrier height changes induced by the $\mathrm{Cu}$ and $\mathrm{Ag}$ adatoms adsorption on $\mathrm{Gr} / \mathrm{H}-\mathrm{Si}(111)$ structures in order to check that the slope parameter $S$ is close to 1 for $\mathrm{Gr} / \mathrm{H}-\mathrm{Si}$ system. DFT calculations have been performed with metal adatoms located on top sites of graphene. We observe that the adsorption of gold adatoms causing a $p$-doping of the graphene sheet moves the Fermi level below the VBM at the Gr/H-Si interface (inversion regime). In this case the MIGS model discussed above is no longer valid. Our calculations show that the adsorption of $\mathrm{Cu}$ and $\mathrm{Ag}$ adatoms leads to $n$-doping of the graphene sheet and that the Fermi level lies in the silicon bandgap at the interface. The electronic and the structural properties of graphene/H-Si(111) are not significantly disturbed by the metal adsorption, only rigid energy shifts of PDOS's are observed. In Fig. 13 is plotted the evolution of the Schottky barrier height variation $\Delta \phi_{B n}$ as a function of $E_{D F}=E_{D}-E_{F}$. The variation is relative to the $\mathrm{SBH}$ value obtained on the bare $\mathrm{Gr} / \mathrm{H}-\mathrm{Si}(111)$ system. As expected the points are aligned on a straight line with a slope in absolute value (obtained by a linear curve fitting of the calculated data) of $0.92 \pm 0.05$ confirming that the Gr/H-Si(111) system approaches the Schottky-Mott limit.

The analysis of the DFT calculation results allows us to conclude that the slope parameter for the $\mathrm{Gr} / \mathrm{H}-\mathrm{Si}(111)$ can be approximated by Eq. (6). In fact, the variation of the Schottky barrier height $\left(\Delta \phi_{B n}\right)$ and the Fermi level energy shift $\left(\Delta E_{F}\right)$ upon the metal deposition on graphene are experimentally accessible using x-ray photoemission spectroscopy. The $\Delta \phi_{B n}$ quantity corresponds to opposite of the Si $2 p$ core-level binding 
energy shift and the Fermi level position change in graphene is given by the $\mathrm{C} 1 \mathrm{~s}$ core-level binding energy shift. Thus the experimental slope parameter can be obtained using the following relation:

$$
S \approx \Delta E_{B}(\operatorname{Si} 2 p) / \Delta E_{B}(\mathrm{C} 1 s)
$$

with $\Delta E_{B}(\operatorname{Si} 2 p)\left(\Delta E_{B}(\mathrm{C} 1 s)\right)$ the binding energy shift of the Si $2 p_{3 / 2}(\mathrm{C} 1 s)$ core-level upon metal deposition. From our experimental data we obtain $S \sim 0.87 \pm 0.07$ that is close to the value deduced from our theoretical calculation. This confirms that the $\mathrm{Gr} / \mathrm{H}-\mathrm{Si}$ system obeys the Schottky-Mott rule.

Our experimental results indicate that the silicon remains in the depletion regime for the adsorption of $\mathrm{Au}$ adatoms on $\mathrm{Gr} / \mathrm{H}-\mathrm{Si}$ structure while the DFT calculations predict that $\mathrm{Au}$ adsorption on graphene results in inversion for silicon (for an $n$-type semiconductor). In addition, the Fermi level position in graphene is experimentally found $\sim 0.29 \mathrm{eV}$ below the Dirac point immediately after transfer on hydrogen-terminated silicon surface showing that the graphene is strongly $p$-doped. These differences between experiment and theory can be explained by the fact that in the real system residual molecules or atoms that impact the work function of graphene or modify the $\mathrm{Si}-\mathrm{H}$ dipole at the hydrogen-terminated silicon are present on the different surfaces. At this stage we have too little information about this point, that's why the possible presence of surface and interface species was neglected in our DFT theoretical approach. It may, however, be noted that the discussion of the slope parameter through MIGS model remains relevant as long as the semiconductor is in depletion regime and that the residual molecules or others species do not modify the density of MIGS in the semiconductor bandgap or the electronic structure of graphene.

A very interesting point emerging from our study is that the MIGS density for the $\mathrm{Gr} / \mathrm{H}-\mathrm{Si}(111)$ interface is much lower than that calculated for the $\mathrm{Au} / \mathrm{H}-\mathrm{Si}(111)$ interface [Table II]. The three order of magnitudes difference between both systems cannot be 
explained by the difference in the density of states at the Fermi energy between graphene and gold layers. In order to identify the origin of this noticeable fact we have performed additional DFT calculations in which the MIGS density at the semiconductor midgap was calculated for various distances between the metal or semimetal and the semiconductor. We have intercalated an interfacial vacuum layer of thickness $t_{\text {vac }}$ between the electrode and the hydrogenated-silicon surface and calculated the electronic structure of the system without geometry optimization. In Fig. 14(a) and (b) are plotted in semilogarithmic scale the evolution of the MIGS density for four $\mathrm{Au}$ layers/H-Si(111) and for $\mathrm{Gr} / \mathrm{H}-\mathrm{Si}(111)$, respectively, as a function of $t_{\mathrm{vac}}$. The two curves show a linear behavior indicating that the MIGS density exponentially decays while moving the metal layers away from the H-Si(111) surface. It can be observed that the exponential decay length $\left(\lambda_{T}\right)$ differs from one structure to the other. In particular we observe that the decay length is about twice lower with graphene $\left(\lambda_{T} \sim 0.28 \AA\right.$ ) than with gold $\left(\lambda_{T} \sim 0.51 \AA\right)$.

Usually for a semi-infinite metal in intimate contact with a semiconductor, free electron metal Bloch waves tunnel into the semiconductor bandgap. When the metal is moved away from the semiconductor surface a vacuum gap is introduced between the two materials. The two systems are progressively decoupled and Bloch waves exponentially decay in the vacuum tunnel barrier. The wave functions will fall exponentially as a function of $t_{\mathrm{vac}}$ as $\sim \exp \left[-\kappa t_{\mathrm{vac}}\right]$, with $\kappa$ the decay constant of the wave function in the barrier. In order to estimate the value of $\kappa$ we assume that the vacuum tunnel barrier is rectangular, that the height of the energy barrier corresponds to the value of the metal work function $\Phi_{m}$ and that the electrons wave vectors are perpendicular to the interfaces. Using a one-dimensional free electron model the decay constant of the wave functions expresses as: 


$$
\kappa=\sqrt{\frac{2 m_{0} \Phi_{m}}{\hbar^{2}}},
$$

with $m_{0}$ the free electron mass and $\hbar$ the reduced Planck's constant. Since the decay length of the wave function in the barrier is given by $1 / \kappa$, the decay length of the density of state through the vacuum barrier is $1 /(2 \kappa)$. Taking $\Phi_{m}=4.0 \mathrm{eV}$ we estimate that the MIGS density at the midgap of semiconductor must exponentially decay with a length of $0.49 \AA$ when the metal is moved away from the semiconductor surface. This value is close to the value obtained from the DFT calculations for the $\mathrm{Au} / \mathrm{H}-\mathrm{Si}(111)$ junction and validate our model.

We have observed that in $\mathrm{Gr} / \mathrm{H}-\mathrm{Si}(111)$ structure the graphene sheet is mainly decoupled from the semiconductor. The electronic band structure of graphene is then well defined in the plane. In contrast, this two-dimensional system does not have propagating character in the direction perpendicular to the sheet. However, the electron wave functions in graphene can be written as a product of in-plane Bloch functions and an one-dimensional function that exponentially decays in the out-of-plane direction [90-92]. We assume that this out-of-plane wave-function decay as $\sim \exp \left[-\kappa^{\prime} t_{\text {vac }}\right]$ in the vacuum barrier separating the semiconductor and the graphene sheet. The decay constant $\kappa^{\prime}$ obtained using a threedimensional model is given by the following relation $[91,92]$ :

$$
\kappa^{\prime}=\sqrt{\frac{2 m_{0} \Phi_{\mathrm{Gr}}}{\hbar^{2}}+k_{/ /}^{2}},
$$

with $k_{/ /}=4 \pi /\left(3 a_{\mathrm{Gr}}\right)$ the parallel momentum taken at the Dirac points [1]. Taking $\Phi_{\mathrm{Gr}}=4.0 \mathrm{eV}$, the exponential decay length $\lambda_{T}=1 /\left(2 \kappa^{\prime}\right)$ is estimated to be $0.25 \AA$, a value very close to that obtained from the DFT calculations. The $k_{/ /}^{2}$ term appears as a dominant term under the square root in the Eq. (9). It follows that $\lambda_{T}$ can be approximated by $1 /\left(2\left|k_{/ /}\right|\right)$ 
demonstrating that $\lambda_{T}$ is mainly controlled by the magnitude of the parallel momentum at the Dirac points. The low MIGS density at the Gr/H-Si(111) interface is therefore clearly related to the two-dimensional nature of the graphene layer. We think that this finding brings precious information to understand the mechanisms governing the formation and the electronic properties of Schottky barriers at 2D-metal/3D-semiconductor interfaces.

\section{CONCLUSION}

By combining XPS experiments and DFT calculations we have studied the mechanisms governing the formation of Schottky barriers at graphene/hydrogen-passivated silicon interface where the graphene plays the role of a $2 \mathrm{D}$ metal electrode. To control the graphene work function without altering neither the structure nor the band dispersion of graphene we have used a method that consists in depositing small amounts of gold forming clusters on $\mathrm{Gr} / \mathrm{H}-\mathrm{Si}$ system under UHV environment. We observe from XPS experiments that the Fermi level is mainly free from pinning at the $\mathrm{Gr} / \mathrm{H}-\mathrm{Si}(001)$ interface demonstrating that the states density in the semiconductor bandgap at the interface is low (typically below $10^{12}$ states $/ \mathrm{cm}^{2}$ ). These results are in agreement with the DFT calculations that, in addition, show that the graphene layer and the semiconductor are decoupled and that the MIGS density at the silicon midgap at interface is very low $\left(<5 \times 10^{10}\right.$ states $\left./ \mathrm{cm}^{2}\right)$. A very important conclusion that stems from these works is that the MIGS density at the semiconductor midgap is low owing to the two-dimensional nature of the graphene layer. We think that these findings bring precious information for the development of 2D-metal/3D-semiconductorbased devices relevant for future applications in electronics, optoelectronics, and photovoltaics.

\section{ACKNOWLEDGMENTS}

This work was supported by the French National Research Agency (ANR) FEOrgSpin Project (Grant Nos. ANR-18-CE24-0017-04) and ENSEMBLE project (Grant Nos. ANR-14- 
CE26-0028-03). The authors warmly acknowledge A. Le Pottier, G. Raffy, and J. Gardais for the technical support. 


\section{References}

[1] A. H. Castro Neto, F. Guinea, N. M. R. Peres, K. S. Novoselov, and A. K. Geim, Rev. Mod. Phys. 81, 109 (2009).

[2] X. Li, Y. Zhu, W. Cai, M. Borysiak, B. Han, D. Chen, R. D. Piner, L. Colombo, and R. S. Ruoff, Nano Lett. 9, 4359 (2009).

[3] J. K. Wassei and R. B. Kaner, Materials Today 13, 52 (2010).

[4] S. Kim, J. Nah, I. Jo, D. Shahrjerdi, L. Colombo, Z. Yao, E. Tutuc, and S. K. Banerjee, Appl. Phys. Lett. 94, 062107 (2009).

[5] X. Li, H. Zhu, K. Wang, A. Cao, J. Wei, C. Li, Y. Jia, Z. Li, X. Li, and D. Wu, Adv. Mater. 22, $2743(2010)$.

[6] K. Kim, J.-Y. Choi, T. Kim, S.-H. Cho, and H.-J. Chung, Nature 479, 338 (2011).

[7] K. S. Novoselov, V. I. Fal'ko, L. Colombo, P. R. Gellert, M. G. Schwab, and K. Kim, Nature 490, 192 (2012).

[8] F. Xia, H. Wang, D. Xiao, M. Dubey, and A. Ramasubramaniam, Nat. Photonics 8, 899 (2014).

[9] C. Xie, Y. Wang, Z.-X. Zhang, D. Wang, and L.-B. Luo, Nano Today 19, 41 (2018).

[10] S. K. Behura, C. Wang, Y. Wen, and V. Berry, Nat. Photonics 13, 312 (2019).

[11] Y. Song, X. Li, C. Mackin, X. Zhang, W. Fang, T. Palacios, H. Zhu, and J. Kong, Nano Lett. 15, $2104(2015)$.

[12] X. Kong, L. Zhang, B. Liu, H. Gao, Y. Zhang, H. Yan, and X. Song, RCS Adv. 9, 863 (2019).

[13] X. An, F. Liu, Y. J. Jung, and S. Kar, Nano Lett. 13, 909 (2013).

[14] M. A. Uddin, A. K. Singh, T. S. Sudarshan, and G. Koley, Nanotechnology 25, 125501 (2014).

[15] D. Schall, D. Neumaier, M. Mohsin, B. Chmielak, J. Bolten, C. Porschatis, A. Prinzen, C. Matheisen, W. Kuebart, B. Junginger, W. Templ, A. L. Giesecke, and H. Kurz, ACS Photonics 1, 781 (2014).

[16] X. Li, M. Zhu, M. Du, Z. Lv, L. Zhang, Y. Li, Y. Yang, T. Yang, X. Li, K. Wang, H. Zhu, and Y. Fang, Small 12, 595 (2016).

[17] N. Unsuree, H. Selvi, M. G. Crabb, J. A. Alanis, P. Parkinson, and T. J. Echtermeyer, 2D Mater. 6, 041004 (2019).

[18] H. Yang, J. Heo, S. Park, H. J. Song, D. H. Seo, K.-E. Byun, P. Kim, I. Yoo, H.-J. Chung, and K. Kim, Science 336, 1140 (2012).

[19] A. Di Bartolomeo, Phys. Rep. 606, 1 (2016).

[20] A. D. Bartolomeo, G. Luongo, F. Giubileo, N. Funicello, G. Niu, T. Schroeder, M. Lisker, and G. Lupina, 2D Mater. 4, 025075 (2017).

[21] A. Alnuaimi, I. Almansouri, I. Saadat, and A. Nayfeh, RSC Adv. 8, 10593 (2018).

[22] N. H. Vu, H. Van Le, T. B. Phan, T. T. Nguyen, N. Thoai, and T. M. Cao, J. Phys. Chem. C 124, $8958(2020)$.

[23] V. Heine, Phys. Rev. 138, A1689 (1965).

[24] J. Tersoff, Phys. Rev. Lett. 52, 465 (1984).

[25] J. Tersoff, Surf. Sci. 168, 275 (1986).

[26] S. G. Louie, J. R. Chelikowsky, and M. L. Cohen, Phys. Rev. B 15, 2154 (1977).

[27] W. Mönch, J. Vac. Sci. Technol. B 17, 1867 (1999).

[28] Y.-J. Lin and J.-J. Zeng, Appl. Surf. Sci. 322, 225 (2014).

[29] J.-C. Le Breton, S. Tricot, G. Delhaye, B. Lépine, P. Turban, and P. Schieffer, Appl. Phys. Lett. 109, 051601 (2016).

[30] J. Courtin, S. Le Gall, P. Chrétien, A. Moréac, G. Delhaye, B. Lépine, S. Tricot, P. Turban, P. Schieffer, and J.-C. Le Breton, Nanoscale Adv. 1, 3372 (2019).

[31] E. Yablonovitch, D. L. Allara, C. C. Chang, T. Gmitter, and T. B. Bright, Phys. Rev. Lett. 57, 249 (1986).

[32] G. S. Higashi, Y. J. Chabal, G. W. Trucks, and K. Raghavachari, Appl. Phys. Lett. 56, 656 (1990).

[33] G. W. Trucks, K. Raghavachari, G. S. Higashi, and Y. J. Chabal, Phys. Rev. Lett. 65, 504 (1990).

[34] K. Pi, K. M. McCreary, W. Bao, W. Han, Y. F. Chiang, Y. Li, S.-W. Tsai, C. N. Lau, and R. K. Kawakami, Phys. Rev. B 80, 075406 (2009). 
[35] P. H. Citrin, G. K. Wertheim, and Y. Baer, Phys. Rev. B 27, 3160 (1983).

[36] J. J. Mortensen, L. B. Hansen, and K. W. Jacobsen, Phys. Rev. B 71, 035109 (2005).

[37] J. Enkovaara, C. Rostgaard, J. J. Mortensen, J. Chen, M. Dułak, L. Ferrighi, J. Gavnholt, C. Glinsvad, V. Haikola, H. A. Hansen, H. H. Kristoffersen, M. Kuisma, A. H. Larsen, L. Lehtovaara, M. Ljungberg, O. Lopez-Acevedo, P. G. Moses, J. Ojanen, T. Olsen, V. Petzold, N. A. Romero, J. Stausholm-Møller, M. Strange, G. A. Tritsaris, M. Vanin, M. Walter, B. Hammer, H. Häkkinen, G. K. H. Madsen, R. M. Nieminen, J. K. Nørskov, M. Puska, T. T. Rantala, J. Schiøtz, K. S. Thygesen, and K. W. Jacobsen, J. Phys. Condens. Matter 22, 253202 (2010).

[38] J. P. Perdew, K. Burke, and M. Ernzerhof, Phys. Rev. Lett. 77, 3865 (1996).

[39] H. J. Monkhorst and J. D. Pack, Phys. Rev. B 13, 5188 (1976).

[40] W. Tang, E. Sanville, and G. Henkelman, J. Phys. Condens. Matter 21, 084204 (2009).

[41] F. J. Himpsel, F. R. McFeely, A. Taleb-Ibrahimi, J. A. Yarmoff, and G. Hollinger, Phys. Rev. B 38, 6084 (1988).

[42] H. D. Barber, Solid-State Electron. 10, 1039 (1967).

[43] F. J. Himpsel, G. Hollinger, and R. A. Pollak, Phys. Rev. B 28, 7014 (1983).

[44] Y. Takata, Y. Kayanuma, M. Yabashi, K. Tamasaku, Y. Nishino, D. Miwa, Y. Harada, K. Horiba, S. Shin, S. Tanaka, E. Ikenaga, K. Kobayashi, Y. Senba, H. Ohashi, and T. Ishikawa, Phys. Rev. B 75, 233404 (2007).

[45] D. Ferrah, O. Renault, C. Petit Etienne, H. Okuno, C. Berne, V. Bouchiat, and G. Cunge, Surf. Interface Anal. 48, 451 (2016).

[46] Y.-C. Lin, C.-C. Lu, C.-H. Yeh, C. Jin, K. Suenaga, and P.-W. Chiu, Nano Lett. 12, 414 (2012).

[47] G. Cunge, D. Ferrah, C. Petit-Etienne, A. Davydova, H. Okuno, D. Kalita, V. Bouchiat, and O. Renault, J. Appl. Phys. 118, 123302 (2015).

[48] C.-Y. Lin, H.-W. Shiu, L.-Y. Chang, C.-H. Chen, C.-S. Chang, and F. S.-S. Chien, J. Phys. Chem. C 118, 24898 (2014).

[49] G. Giovannetti, P. A. Khomyakov, G. Brocks, V. M. Karpan, J. van den Brink, and P. J. Kelly, Phys. Rev. Lett. 101, 026803 (2008).

[50] P. A. Khomyakov, G. Giovannetti, P. C. Rusu, G. Brocks, J. van den Brink, and P. J. Kelly, Phys. Rev. B 79, 195425 (2009).

[51] C. Gong, G. Lee, B. Shan, E. M. Vogel, R. M. Wallace, and K. Cho, J. Appl. Phys. 108, 123711 (2010).

[52] A. Varykhalov, M. R. Scholz, T. K. Kim, and O. Rader, Phys. Rev. B 82, 121101(R) (2010).

[53] K. M. McCreary, K. Pi, A. G. Swartz, W. Han, W. Bao, C. N. Lau, F. Guinea, M. I. Katsnelson, and R. K. Kawakami, Phys. Rev. B 81, 115453 (2010).

[54] Z. Zhou, F. Gao, and D. W. Goodman, Surf. Sci. 604, L31 (2010).

[55] L. Liu, Z. Chen, L. Wang, E. Polyakova (Stolyarova), T. Taniguchi, K. Watanabe, J. Hone, G. W. Flynn, and L. E. Brus, J. Phys. Chem. B 117, 4305 (2013).

[56] L. Hu, X. Hu, X. Wu, C. Du, Y. Dai, and J. Deng, Physica B Condens. Matter 405, 3337 (2010).

[57] K. T. Chan, J. B. Neaton, and M. L. Cohen, Phys. Rev. B 77, 235430 (2008).

[58] M. Amft, B. Sanyal, O. Eriksson, and N. V. Skorodumova, J. Phys. Condens. Matter 23, 205301 (2011).

[59] J. Ding, Z. Qiao, W. Feng, Y. Yao, and Q. Niu, Phys. Rev. B 84, 195444 (2011).

[60] I. Gierz, C. Riedl, U. Starke, C. R. Ast, and K. Kern, Nano Lett. 8, 4603 (2008).

[61] Z. Klusek, P. Dabrowski, P. Kowalczyk, W. Kozlowski, W. Olejniczak, P. Blake, M. Szybowicz, and T. Runka, Appl. Phys. Lett. 95, 113114 (2009).

[62] M. Vanin, J. J. Mortensen, A. K. Kelkkanen, J. M. Garcia-Lastra, K. S. Thygesen, and K. W. Jacobsen, Phys. Rev. B 81, 081408(R) (2010).

[63] M. K. Srivastava, Y. Wang, A. F. Kemper, and H.-P. Cheng, Phys. Rev. B 85, 165444 (2012).

[64] X. Liu, C. Z. Wang, M. Hupalo, W. C. Lu, M. C. Tringides, Y. X. Yao, and K. M. Ho, Phys. Chem. Chem. Phys. 14, 9157 (2012).

[65] M. Manadé, F. Viñes, and F. Illas, Carbon 95, 525 (2015).

[66] R. M. Del Castillo and L. E. Sansores, Eur. Phys. J. B 88, 248 (2015).

[67] C. Gong, G. Lee, B. Shan, E. M. Vogel, R. M. Wallace, and K. Cho, J. Appl. Phys. 108, 123711 (2010).

[68] P. V. C. Medeiros, G. K. Gueorguiev, and S. Stafström, Phys. Rev. B 85, 205423 (2012). 
[69] V. Barone, J. E. Peralta, J. Uddin, and G. E. Scuseria, J. Chem. Phys. 124, 024709 (2006).

[70] M. Legesse, F. E. Mellouhi, E. T. Bentria, M. E. Madjet, T. S. Fisher, S. Kais, and F. H. Alharbi, Appl. Surf. Sci. 394, 98 (2017).

[71] X. Liu, Z. Zhang, Z. Luo, B. Lv, and Z. Ding, Nanomaterials 9, 1674 (2019).

[72] N. Zhao, L. Xu, H.-Y. Hsu, T.-C. Leung, and M.-C. Lin, J. Vac. Sci. Technol. B 38, 022211 (2020).

[73] H. Fukutome, K. Takano, H. Yasuda, K. Maehashi, S. Hasegawa, and H. Nakashima, Appl. Surf. Sci. 130-132, 346 (1998).

[74] M. Buongiorno Nardelli, F. Finocchi, M. Palummo, R. Di Felice, C. M. Bertoni, F. Bernardini, and S. Ossicini, Surf. Sci. 269-270, 879 (1992).

[75] F. Cargnoni, C. Gatti, E. May, and D. Narducci, J. Chem. Phys. 112, 887 (1999).

[76] M. Sajjad, X. Yang, P. Altermatt, N. Singh, U. Schwingenschlögl, and S. De Wolf, Appl. Phys. Lett. 114, 071601 (2019).

[77] X. Dang, H. Dong, L. Wang, Y. Zhao, Z. Guo, T. Hou, Y. Li, and S.-T. Lee, ACS Nano 9, 8562 (2015).

[78] Y. Gohda, S. Watanabe, and A. Groß, Phys. Rev. Lett. 101, 166801 (2008).

[79] W. Bludau, A. Onton, and W. Heinke, J. Appl. Phys. 45, 1846 (1974).

[80] C. S. Wang and W. E. Pickett, Phys. Rev. Lett. 51, 597 (1983).

[81] Á. Morales-García, R. Valero, and F. Illas, J. Phys. Chem. C 121, 18862 (2017).

[82] H. Lu, Y. Guo, H. Li, and J. Robertson, Appl. Phys. Lett. 114, 222106 (2019).

[83] W. Mönch, Rep. Prog. Phys. 53, 221 (1990).

[84] A. M. Cowley and S. M. Sze, J. Appl. Phys. 36, 3212 (1965).

[85] S. G. Louie, J. R. Chelikowsky, and M. L. Cohen, J. Vac. Sci. Technol. 13, 790 (1976).

[86] T. Fang, A. Konar, H. Xing, and D. Jena, Appl. Phys. Lett. 91, 092109 (2007).

[87] F. Guinea, Phys. Rev. B 75, 235433 (2007).

[88] M. A. Kuroda, J. Tersoff, and G. J. Martyna, Phys. Rev. Lett. 106, 116804 (2011).

[89] H. Rokni and W. Lu, Sci. Rep. 7, 42821 (2017).

[90] L. Britnell, R. V. Gorbachev, A. K. Geim, L. A. Ponomarenko, A. Mishchenko, M. T. Greenaway, T. M. Fromhold, K. S. Novoselov, and L. Eaves, Nat. Commun. 4, 1794 (2013).

[91] R. M. Feenstra, D. Jena, and G. Gu, J. Appl. Phys. 111, 043711 (2012).

[92] F. A. Chaves, D. Jiménez, A. W. Cummings, and S. Roche, J. Appl. Phys. 115, 164513 (2014). 
Tables

TABLE I. Adsorption energy $\left(E_{\text {ads }}\right)$, vertical equilibrium Au-graphene distance $\left(d_{\mathrm{M}-\mathrm{Gr}}\right)$ and gold charge ( $Q_{\mathrm{Au}}$ in atomic units, $q$ is the elementary charge) as obtained through a Bader analysis for Au adsorption onto hollow, bridge, and top sites of pristine graphene.

\begin{tabular}{lccc}
\hline \hline Adsorption site & $E_{\mathrm{ads}}(\mathrm{eV})$ & $d_{\mathrm{M}-\mathrm{Gr}}(\AA)$ & $Q_{\mathrm{Au}}(q)$ \\
\hline Top & 0.130 & 2.52 & -0.07 \\
Bridge & 0.136 & 3.41 & -0.10 \\
Hollow & 0.130 & 3.62 & -0.10 \\
\hline \hline
\end{tabular}

TABLE II. Equilibrium distance between the metal interface layer and the first silicon layer ( $\left.d_{\mathrm{M}-\mathrm{Si}}\right)$, MIGS exponential decay length $\left(\lambda_{\mathrm{MIGS}}\right)$, density of MIGS $\left(D_{\mathrm{MIGS}}\right)$ at the semiconductor midgap, metal Thomas-Fermi screening length $\left(\delta_{m}\right)$ and slope parameter $S$ for the various considered systems. The distance $d_{\mathrm{M}-\mathrm{Si}}$ corresponds to the average distance in $z$ coordinate between the position of the gold or carbon atoms in the interface plane and the position of the first silicon layer.

\begin{tabular}{lccccc}
\hline \hline & $d_{\mathrm{M}-\mathrm{Si}}(\AA)$ & $\lambda_{\text {MIGS }}(\AA)$ & $D_{\text {MIGS }}\left(\right.$ states $\left./ \mathrm{eV} / \mathrm{cm}^{2}\right)$ & $\delta_{m}(\AA)$ & $S$ \\
\hline $\mathrm{Au} / \mathrm{Si}(111)$ & 2.26 & 2.8 & $2.4 \times 10^{14}$ & 0.5 & 0.1 \\
$\mathrm{Au} / \mathrm{H}-\mathrm{Si}(111)$ & 3.88 & 2.7 & $3.7 \times 10^{13}$ & 0.5 & 0.4 \\
$\mathrm{Gr} / \mathrm{H}-\mathrm{Si}(111)$ & 4.62 & 3.6 & $4.3 \times 10^{10}$ & $0.6-4$ & 1.0 \\
\hline \hline
\end{tabular}


Figure captions

FIG. 1. (a) Si $2 p$ core-level experimental spectra and line shape decomposition for various $\mathrm{Au}$ thicknesses deposited on the $\mathrm{Gr} / \mathrm{H}-\mathrm{Si}(001)$ surface. The experimental spectra were collected at normal emission and at a photon energy of $1253.6 \mathrm{eV}$. The component labelled $\mathrm{Si}^{0}\left(\mathrm{Si}^{1+}\right)$ is assigned to an electron emission from the $\mathrm{Si}$ substrate $\left(\mathrm{Si}_{2} \mathrm{O}\right.$ patches at the $\mathrm{Si}$ surface). (b) Evolution of the Schottky barrier height $\left(\phi_{B n}\right)$ as a function of the Au thickness. The data were obtained from the position of the Si $2 p_{3 / 2}$ core-level position of the main component.

FIG. 2. (Color online) (a) C $1 s$ core-level experimental spectra and line shape decomposition for various $\mathrm{Au}$ thicknesses deposited on the $\mathrm{Gr} / \mathrm{H}-\mathrm{Si}(001)$ surface. The experimental spectra were collected at normal emission and at a photon energy of $1253.6 \mathrm{eV}$. The component labelled sp2 is related to carbon atoms connected in a honeycomb lattice structure with sp2 hybridization whereas the component labelled sp3 is related to sp3 hybridized carbon atoms. (b) Evolution of the binding energy of the sp2 component of the $\mathrm{C} 1 \mathrm{~s}$ core-level as a function of the Au thickness.

FIG. 3. Evolution of the binding energy (BE) of the Si $2 p_{3 / 2}$ core-level (main component) as a function of the binding energy of the $\mathrm{C} 1 s$ core-level. The binding energies were obtained from the core-level spectra analysis for the different Au thicknesses. The experimental points are aligned. The linear fit of the dataset (full line) yields a positive slope of $0.87 \pm 0.07$.

FIG. 4. Schematic representation of the three adsorption sites on graphene for the adatoms adsorption considered in our DFT calculations. 
FIG. 5. (Color online) Projected density of states on atomic orbital of carbon atoms for relaxed structures with Au atoms on the top, bridge and hollow sites of graphene and for the pristine graphene.

FIG. 6. Averaged electrostatic potentials over the $x y$-plane as a function of the position along the $z$ direction for the pristine graphene (located at $z=20 \AA$ ) with (dashed line) and without (full line) $\mathrm{Au}$ atom on the top site of graphene. The Fermi level is taken as reference for the two situations. The single vertical arrow indicates the equilibrium position in $z$ coordinate of the $\mathrm{Au}$ atom adsorbed on the right side of the graphene layer. The work function on the left side of the graphene layer is obtained from the difference between the vacuum level and the Fermi level. For the pristine graphene the work function is $4.23 \mathrm{eV}$. The adsorption of $\mathrm{Au}$ atom at right on the graphene layer causes an increasing of the work function of $0.17 \mathrm{eV}$ on the free-side of the graphene layer.

FIG. 7. Evolution of the work function on the free graphene side caused by the Au atoms adsorption (on the other graphene side) as a function of $E_{D}-E_{F}$. The points obtained from the DFT calculation are aligned. The linear fit of the dataset (full line) yields a positive slope of $0.96 \pm 0.03$.

FIG. 8. (Color online) Schematic representation of the silicon slabs used in our DFT calculations. The slabs were modeled with 12 atomic layers (numbered from 1 to 12 in the figure) and an in-plane unit cell parameter set to $3.87 \AA$, derived from the predicted lattice parameter of bulk Si $(5.47 \AA)$. They were passivated with a hydrogen monolayer on both sides. The interplanar atomic distance in the region near the center of the slab are given in the figure. 
FIG. 9. (Color online) (a) Schematic representations of the DFT-optimized structure for the $\mathrm{Gr} / \mathrm{H}-\mathrm{Si}(111)$ system. At left, top view of graphene on the hydrogen-terminated silicon (111) surface. Arrows give the surface supercell used to perform the DFT calculations. At right, side view of the geometry-optimized system. The equilibrium average distance in $z$ coordinate between the position of the carbon atoms and the hydrogen plane (first silicon layer) of 3.11 (4.62) $\AA$ is also given. (b) Projected density of states on atomic orbital of carbon atoms for the free-standing undoped distorted graphene (dashed line) and for the $\mathrm{Gr} / \mathrm{H}-\mathrm{Si}(111)$ structure (full line). The DFT calculations for the free-standing distorted graphene was performed with a lattice parameter of $2.58 \AA$ (the same parameter as for $\mathrm{Gr}$ on $\mathrm{H}-\mathrm{Si}(111)$ ). The two curves are very similar, only a slight shift in energy $(0.07 \mathrm{eV})$ is detectable.

FIG. 10. (Color online) Schematic representations of the DFT-optimized structure for the four layers of $\mathrm{Au}$ on $\mathrm{H}-\mathrm{Si}(111)$ surface. At left, the top view of the slab. The arrows give the surface supercell used to perform the DFT calculations. At right, side view of the geometryoptimized system. The equilibrium average distance in $z$ coordinate between the position of the interfacial $\mathrm{Au}$ layer and the hydrogen plane (first silicon layer) of 2.35 (3.88) $\AA$ is also given.

FIG. 11. (Color online) (a) Layer-resolved projected density of states on atomic orbital of silicon atoms in semilogarithmic representation for the relaxed structure with four Au layers on the H-Si(111) surface. (b) Evolution, in semilogarithmic representation, of the Si-PDOS at the silicon midgap (PDOS midgap $)$ as a function of the depth in silicon $\left(d_{\mathrm{Si}}\right)$ relative to the first silicon layer. The points are mainly aligned and the linear fit of the dataset allows to 
determine the MIGS exponential decay length in the semiconductor midgap that is estimated to be $\sim 0.27 \mathrm{~nm}$.

FIG. 12. (Color online) (a) Layer-resolved projected density of states on atomic orbital of silicon atoms in semilogarithmic representation for the $\mathrm{Gr} / \mathrm{H}-\mathrm{Si}(111)$ relaxed system. (b) Evolution, in semilogarithmic representation, of the Si-PDOS at the silicon midgap $\left(\mathrm{PDOS}_{\text {midgap }}\right)$ as a function of the depth in silicon $\left(d_{\mathrm{Si}}\right)$ relative to the first silicon layer. The curve deduced from our DFT calculations shows oscillations that we are not able to interpret for now. However, the linear fit of the dataset allows to determinate the MIGS exponential decay length in the semiconductor midgap $\left(\lambda_{\text {MIGS }}=0.36 \mathrm{~nm}\right)$.

FIG .13. (Color online) Variation of the Schottky barrier height as a function of $E_{D F}$. This variation is relative to the $\mathrm{SBH}$ value calculated for the bare $\mathrm{Gr} / \mathrm{H}-\mathrm{Si}(111)$ surface and it was calculated for $\mathrm{Cu}$ and $\mathrm{Ag}$ adatoms adsorbed on $\mathrm{Gr} / \mathrm{H}-\mathrm{Si}(111)$ surface on top sites of graphene. The inset shows a schematic representation of the slab used for calculations with the adsorbed adatoms $(\mathrm{Ag}$ or $\mathrm{Cu})$. The linear fit of the dataset (full line) yields a slope of $-0.92 \pm 0.05$.

FIG. 14. MIGS density in semilogarithmic representation as a function of the vacuum thickness $\left(t_{\mathrm{vac}}\right)$ added between the metal or semimetal layer and the semiconductor. (a) is for $\mathrm{Au} / \mathrm{H}-\mathrm{Si}(111)$ system and (b) is for the bare $\mathrm{Gr} / \mathrm{H}-\mathrm{Si}(111)$ system. For the two systems the DFT-calculated points are aligned. The linear fit of the dataset (full lines) yields an exponential decay length about twice less with graphene $\left(\lambda_{T} \sim 0.28 \AA\right)$ than with gold layer ( $\left.\lambda_{T} \sim 0.51 \AA\right)$. 

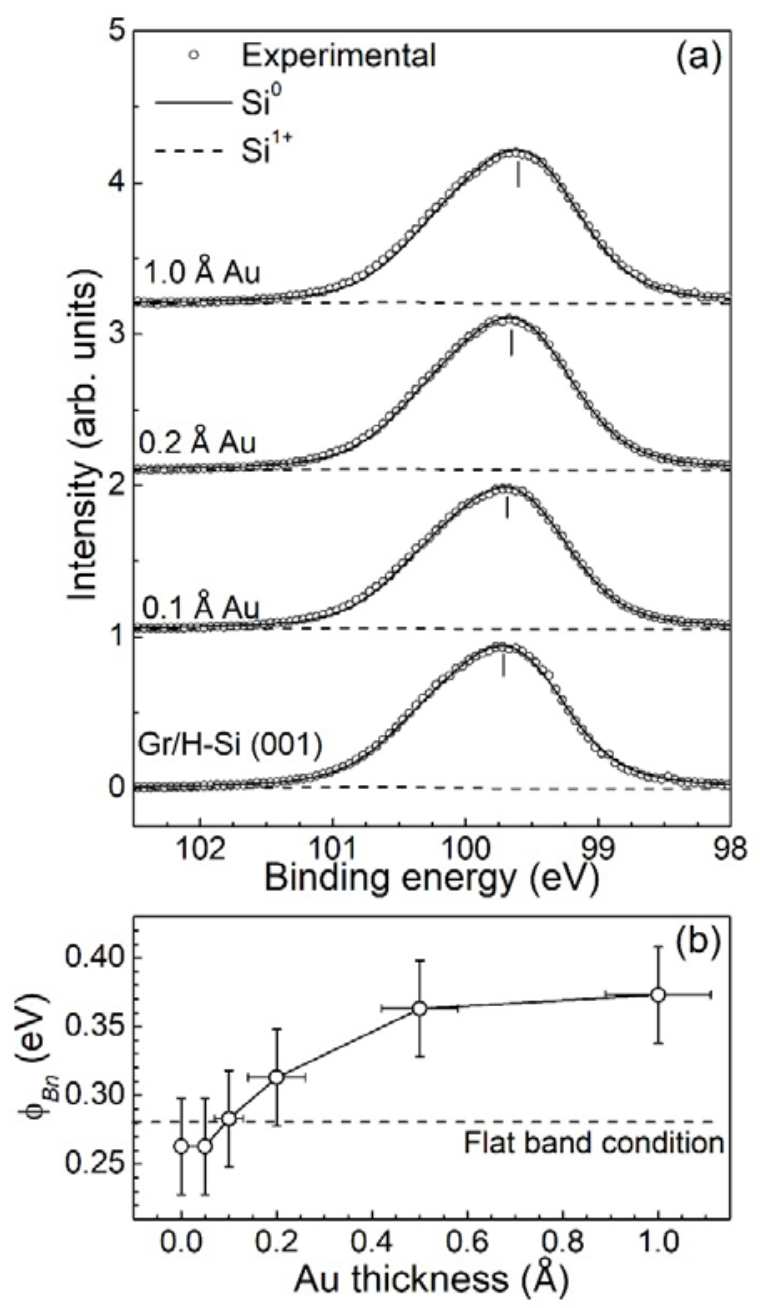

J. Courtin, Figure 1 

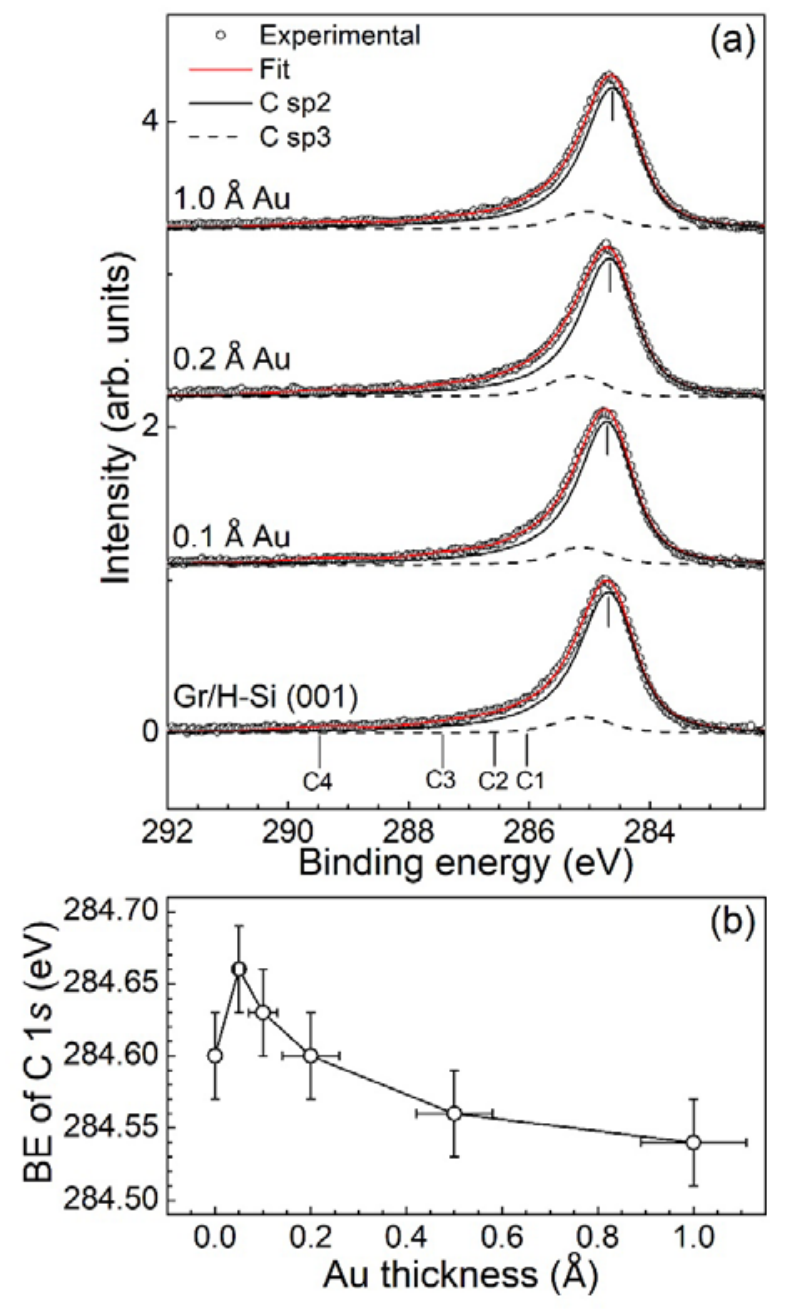

J. Courtin, Figure 2 


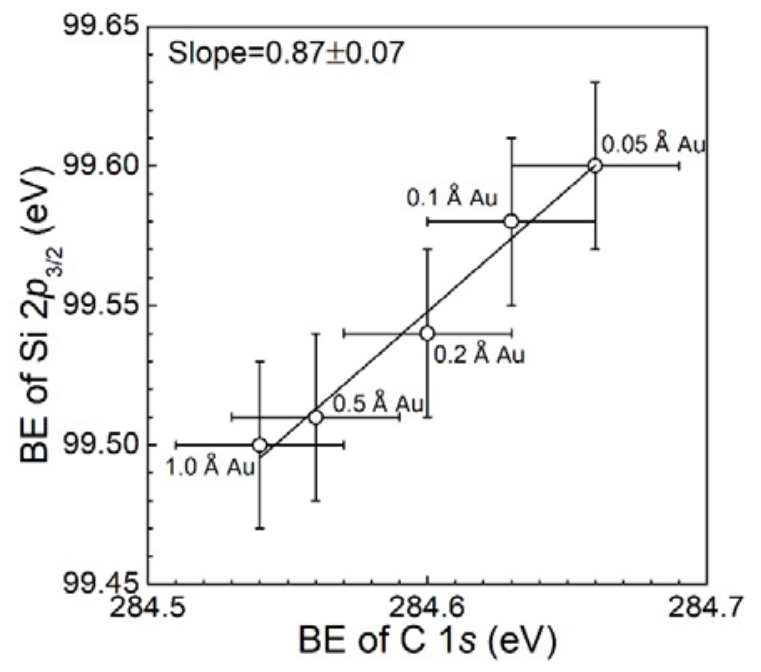

\section{J. Courtin, Figure 3}




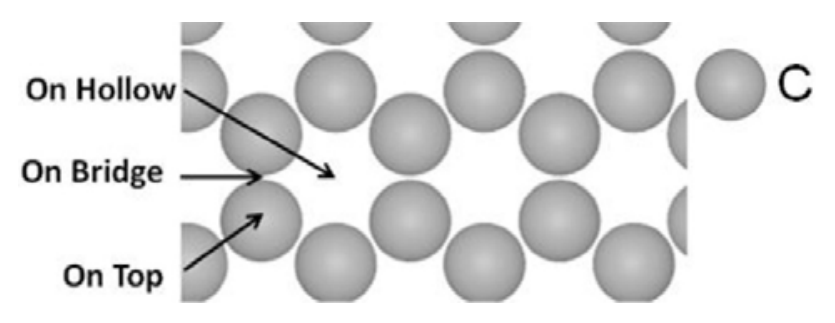

\section{J. Courtin, Figure 4}




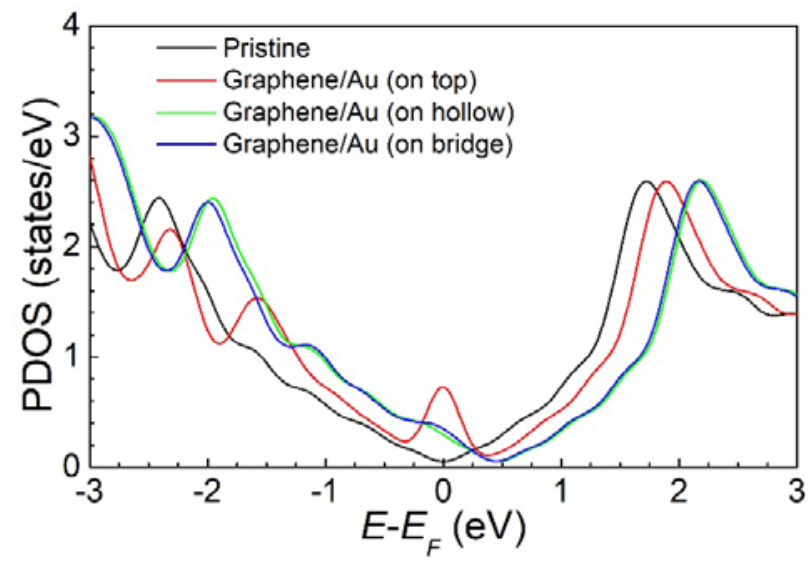

J. Courtin, Figure 5 


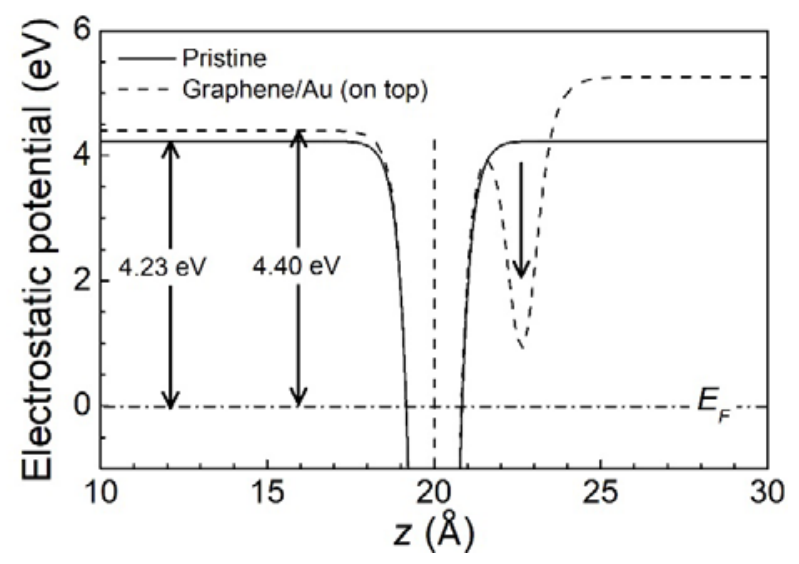

J. Courtin, Figure 6 


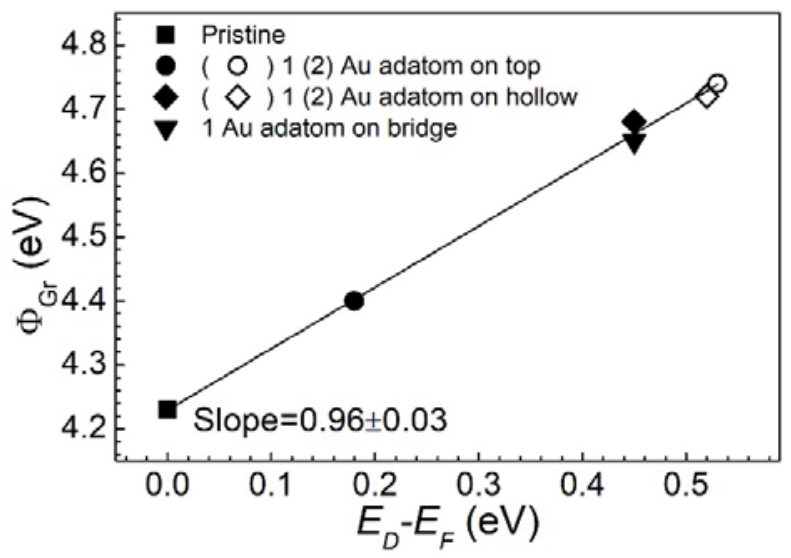

\section{J. Courtin, Figure 7}




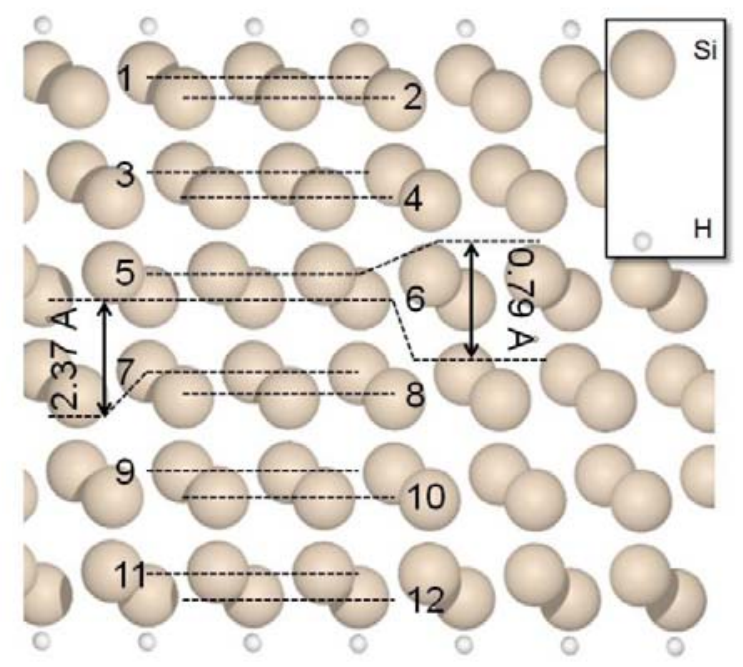

\section{J. Courtin, Figure 8}



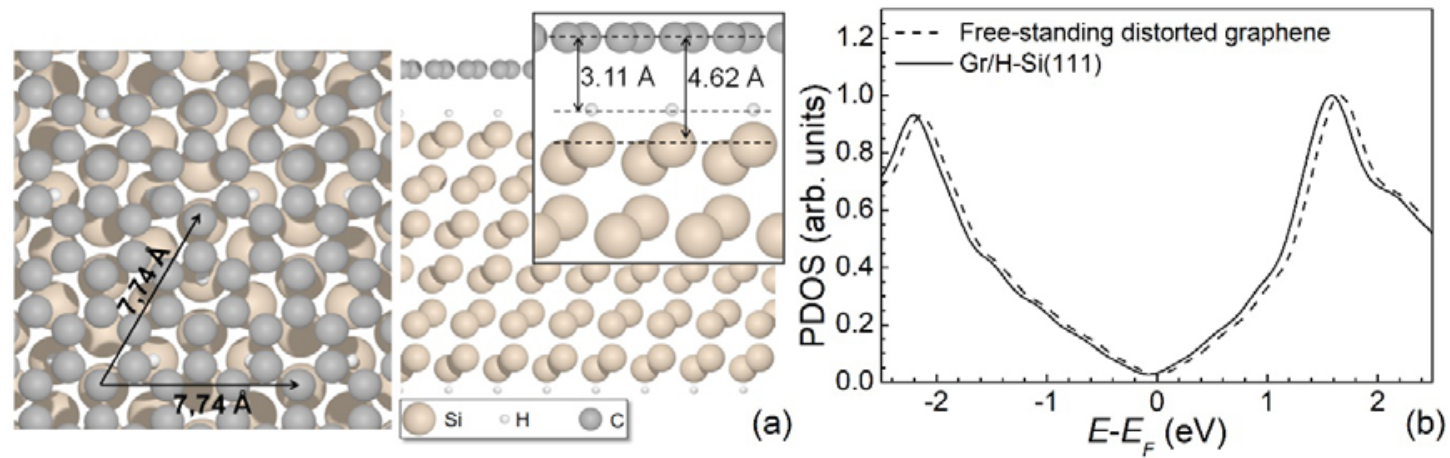

\section{J. Courtin, Figure 9}




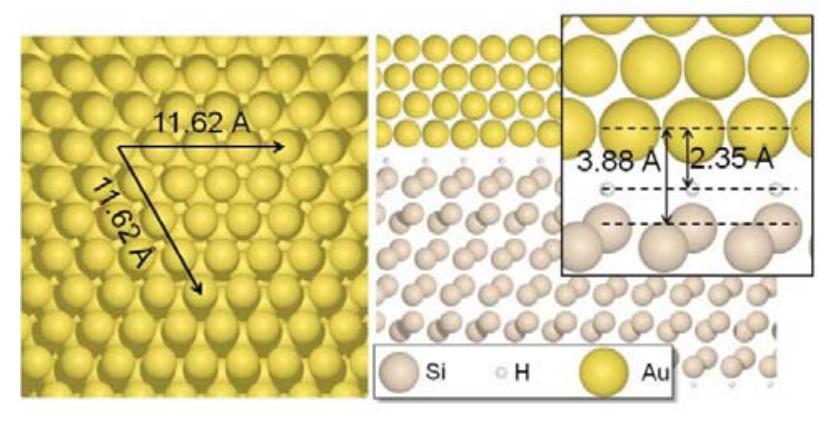

J. Courtin, Figure 10 


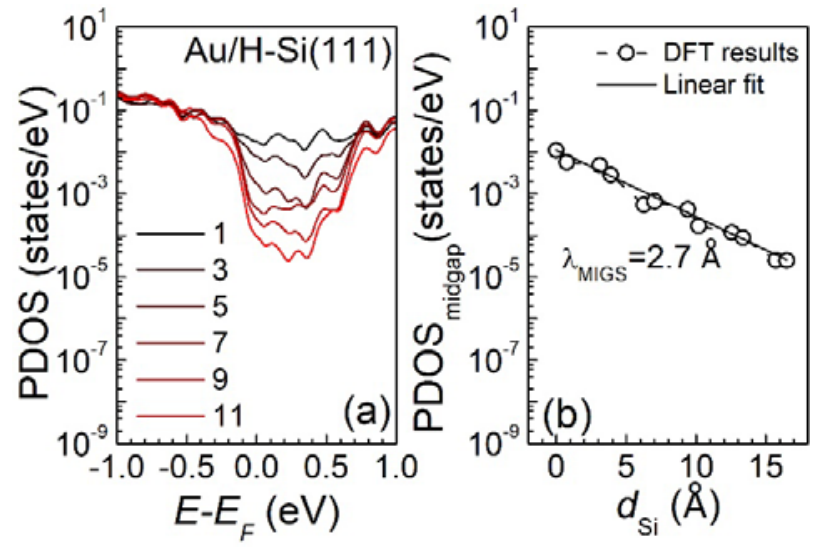

J. Courtin, Figure 11 


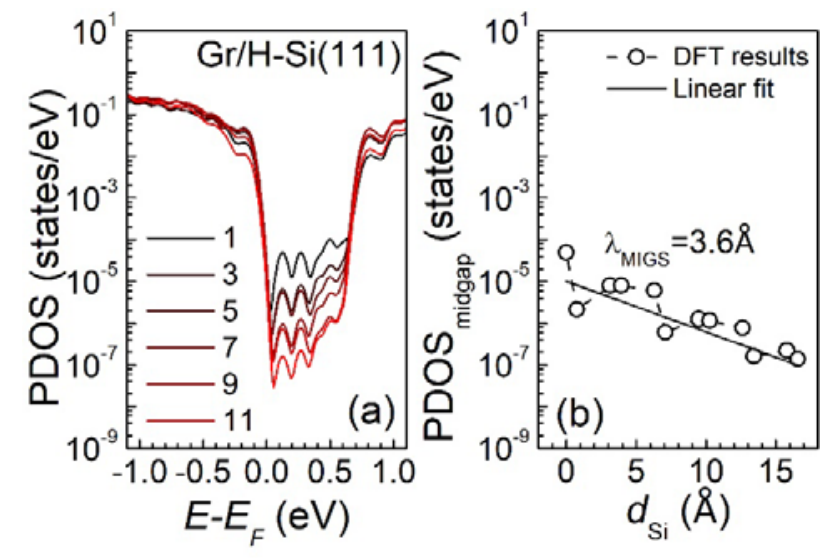

J. Courtin, Figure 12 


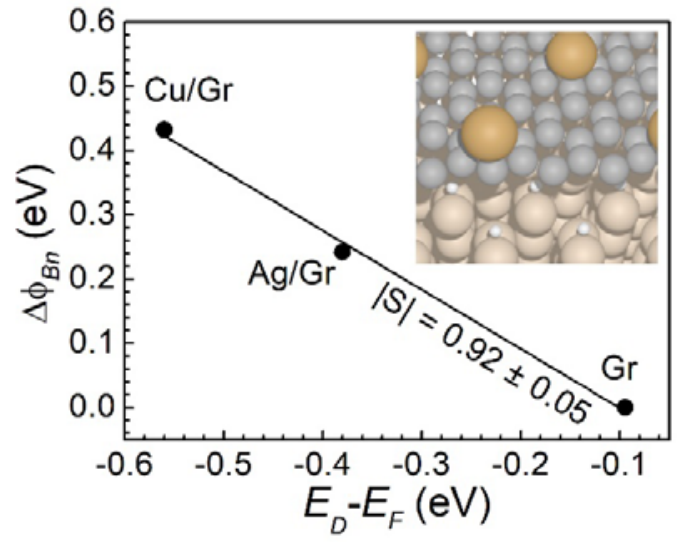

J. Courtin, Figure 13 

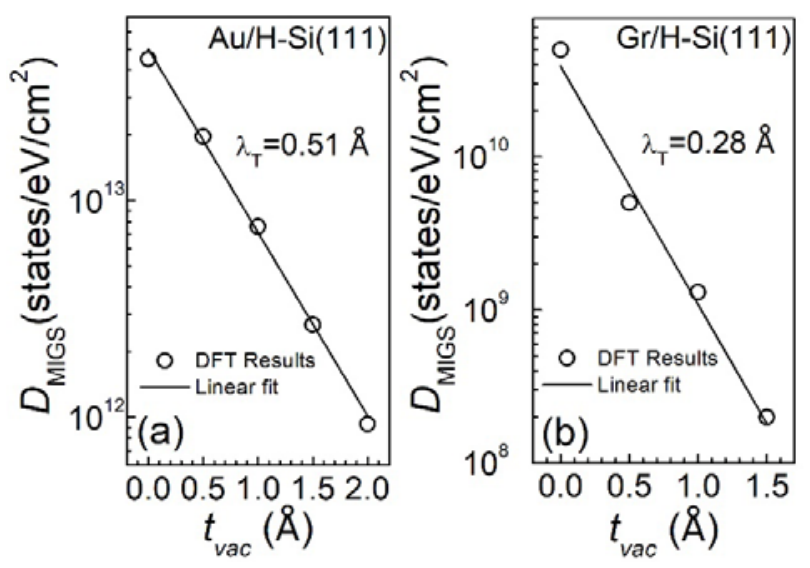

J. Courtin, Figure 14 